IMA Journal of Numerical Analysis (2013) Page 1 of 29

doi:10.1093/imanum/drn000

\title{
Spectral decompositions and nonnormality of boundary integral operators in acoustic scattering
}

\author{
T. Betcke†, J. Phillips $\ddagger$, E. A. SPEnce \\ [Received on 14 January 2013]
}

\begin{abstract}
Understanding the spectral properties of boundary integral operators in acoustic scattering has important practical implications, such as for the analysis of the stability of boundary element discretisations or the convergence of iterative solvers as the wavenumber $k$ grows. Yet little is known about spectral decompositions of the standard boundary integral operators in acoustic scattering. Theoretical results are mainly available on the unit disk, where these operators diagonalise in a simple Fourier basis. In this paper we investigate spectral decompositions for more general smooth domains. Based on the decomposition of the acoustic Green's function in elliptic coordinates we give spectral decompositions on ellipses. For general smooth domains we show that approximate spectral decompositions can be given in terms of circle Fourier modes transplanted onto the boundary of the domain. An important underlying question is whether or not the operators are normal. Based on previous numerical investigations it appears that the standard boundary integral operators are normal only when the domain is a ball and here we prove that this is indeed the case for the acoustic single layer potential. We show that the acoustic single, double and conjugate double layer potential are normal in a scaled inner product on the ellipse. On more general smooth domains the operators can be split into a normal component plus a smooth perturbation. Numerical computations of pseudospectra are presented to demonstrate the nonnonnormal behaviour on general domains.
\end{abstract}

Keywords: acoustic scattering; boundary integral operators; spectra; pseudospectra

\section{Introduction}

The study of spectral properties of partial differential operators has seen tremendous advances in recent decades. An important question is whether an operator is nonnormal and how this nonnormality influences analytical and numerical properties of the operator. The development of pseudospectra as a tool for understanding nonnormality has given great insight into diverse areas, such as existence of solutions to linear PDE problems, the behaviour of numerical solvers, or transient behaviour in dynamical systems. For a wonderful overview about this field we refer to the recent book by Trefethen \& Embree (2005). However, whilst nonnormality has been studied extensively for partial differential operators, little is known for boundary integral operators. The aim of this paper is to study nonnormality in the context of boundary integral operators in acoustic scattering.

Let $\Omega \subset \mathbb{R}^{2}$ be a bounded domain and denote the boundary of $\Omega$ by $\Gamma$. For the majority of this paper we assume that $\Gamma$ is an analytic curve. Denote by $\Omega^{+}:=\mathbb{R}^{2} \backslash \bar{\Omega}$ the exterior of $\Omega$. Furthermore, let $g: \mathbb{R}^{2} \times \mathbb{R}^{2} \rightarrow \mathbb{C}$ be the acoustic Green's function defined by $g(x, y)=\frac{i}{4} H_{0}^{(1)}(k|x-y|)$, where $k$ is the wavenumber and $H_{0}^{(1)}$ denotes the Hankel function of the first kind with order 0 (NIST Digital Library, $\S 10.2)$. We define the following operators, which are bounded operators on $L^{2}(\Gamma)$.

- The acoustic single layer potential

$$
[S \phi](x)=\int_{\Gamma} g(x, y) \phi(y) d s(y), x \in \Gamma
$$

\footnotetext{
†Department of Mathematics, University College London, UK. Corresponding author. Email: t.betcke@ucl.ac.uk. Timo Betcke is supported by Engineering and Physical Sciences Research Council (EPSRC) Grant EP/H004009/1.

Department of Mathematics, University College London, UK. Email: joel.phillips@ucl.ac.uk. Joel Phillips is supported by EPSRC Grant EP/H004009/1.

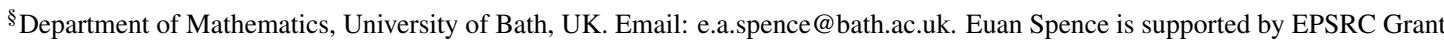
$\mathrm{EP} / 1025995 / 1$.
} 
- The acoustic double layer potential

$$
[K \phi](x)=\frac{\phi(x)}{2}+\int_{\Gamma} \frac{\partial}{\partial n(y)} g(x, y) \phi(y) d s(y), x \in \Gamma
$$

- The acoustic conjugate double layer potential

$$
[T \phi](x)=-\frac{\phi(x)}{2}+\int_{\Gamma} \frac{\partial}{\partial n(x)} g(x, y) \phi(y) d s(y), x \in \Gamma
$$

Here, $n$ denotes the exterior normal to $\Omega$. The double layer and conjugate double layer potentials usually appear without the factors of $\pm 1 / 2$. Our reason for defining them like this is that if we denote the exterior trace operator by $\gamma_{0}^{+}$and the exterior normal derivative by $\gamma_{1}^{+}$, then the above boundary operators are obtained from the single and double layer potentials,

$$
[\mathscr{S} \phi](x)=\int_{\Gamma} g(x, y) \phi(y) d s(y), \quad[\mathscr{K} \phi](x)=\int_{\Gamma} \frac{\partial}{\partial n(y)} g(x, y) \phi(y) d s(y), x \in \Omega^{+}
$$

by $S=\gamma_{0}^{+} \mathscr{S}, K=\gamma_{0}^{+} \mathscr{K}, T=\gamma_{1}^{+} \mathscr{S}$. The fact that $S, K$, and $T$ arise directly as the traces of $\mathscr{S}$ and $\mathscr{K}$ makes the algebra of the spectral decompositions considered below more convenient. For mapping properties of these operators in Sobolev spaces see, for example, McLean (2000). An excellent introduction to integral operators in acoustic scattering is given in the book by Colton \& Kress (1983). We will also be interested in the combined potential operator

$$
A_{\eta}:=2(I+T-i \eta S)
$$

defined for $\eta \neq 0$. In Chandler-Wilde \& Langdon (2007) it was shown that $A_{\eta}$ is a bounded operator with bounded inverse for any $k>0, \eta \neq 0$, mapping $L^{2}(\Gamma)$ into $L^{2}(\Gamma)$ for any Lipschitz boundary $\Gamma$.

The main motivation for studying the behaviour of these integral operators is that they can be used to solve scattering problems modelled by the Helmholtz equation, with the combined potential operator $A_{\eta}$ giving rise to a uniquely-solvable integral equation for the solution of the Helmholtz equation with Dirichlet boundary conditions (see Section 6 for details). Another important boundary integral operator is the hypersingular operator. This is $\gamma_{1}^{+} \mathscr{K}$ and arises when formulating boundary value problems for the Helmholtz equation with Neumann boundary conditions. In this paper we are mainly focused on the behaviour of the combined potential operator $A_{\eta}$ and so we do not consider the hypersingular operator.

The spectral behaviour of the operators $S, K, T$ and $A_{\eta}$ is especially simple on the unit circle. We have the following well known result (see e.g. Kress \& Spassov (1983); Kress (1985); Domínguez et al. (2007)).

THEOREM 1.1 On the unit circle it holds that

$$
S u_{n}=\lambda_{n}^{(S)} u_{n}, K u_{n}=\lambda_{n}^{(K)} u_{n}, T u_{n}=\lambda_{n}^{(T)} u_{n}, A_{\eta} u_{n}=\lambda_{n}^{(A)} u_{n}
$$

where in polar coordinates $u_{n}(\theta)=e^{i n \theta}$ and

$$
\begin{aligned}
\lambda_{n}^{(S)} & =\frac{\pi i}{2} H_{n}^{(1)}(k) J_{n}(k), \\
\lambda_{n}^{(K)} & =\frac{k \pi i}{4} H_{n}^{(1)}(k)\left(J_{n-1}(k)-J_{n+1}(k)\right), \\
\lambda_{n}^{(T)} & =\frac{k \pi i}{4}\left(H_{n-1}^{(1)}(k)-H_{n+1}^{(1)}(k)\right) J_{n}(k) \\
\lambda_{n}^{(A)} & =2\left(1+\lambda_{n}^{(T)}-i \eta \lambda_{n}^{(S)}\right) .
\end{aligned}
$$

Proof. The result is a consequence of the Graf addition formula (NIST Digital Library, eq. 10.23.7), stating that

$$
H_{0}^{(1)}\left(k\left|r e^{i \theta}-\rho e^{i \phi}\right|\right)=\sum_{n=-\infty}^{\infty} e^{i n(\theta-\phi)} H_{n}^{(1)}\left(k r_{>}\right) J_{n}\left(k r_{<}\right)
$$

where $r_{>}=\max (r, \rho)$ and $r_{<}=\min (r, \rho)$. 
(It is well known that on the unit circle $K-\frac{1}{2} I=T+\frac{1}{2} I$ and this can be seen from the eigenvalues using the facts that $J_{n-1}(k)-J_{n+1}(k)=2 J_{n}^{\prime}(k), H_{n-1}^{(1)}(k)-H_{n+1}^{(1)}(k)=2 H_{n}^{(1)^{\prime}}(k)$, and $W\left[J_{n}, H_{n}^{(1)}, k\right]=2 i /(\pi k)$, where $W$ denotes the Wronskian.)

An important feature of the circle case is that the operators $S, K, T$ and $A_{\eta}$ are normal, since they diagonalize in a unitary basis and therefore commute with their adjoints. Recall that a bounded operator $A$ acting on a Hilbert space $\mathscr{H}$ is normal if and only

$$
A A^{*}=A^{*} A,
$$

where $A^{*}$ is the adjoint of $A$. Normal operators are simple in the sense that they can be shown to be unitarily equivalent to multiplication operators (see for example (Conway, 1985, Theorem 4.6)). In particular, if $A$ is compact and normal then there exists an orthonormal system $\left\{\phi_{j}\right\}$, such that

$$
A=\sum_{j} \lambda_{j} \phi_{j}\left\langle\phi_{j}, \cdot\right\rangle
$$

where the $\lambda_{j}$ are the eigenvalues of $A$ and the $\phi_{j}$ are the associated eigenfunctions. Hence, the behaviour of a normal operator can be completely determined by spectral information.

For more general domains than the circle, little is known about spectral decompositions, however understanding the spectral properties and normality of these boundary integral operators has both theoretical and practical implications. On the theoretical side, in Ramm $(1973,1980)$ Ramm asked when the eigensystem of the single layer potential operator in 3-d forms a complete basis in $L^{2}(\Gamma)$ and gave normality as a sufficient condition. On the practical side, whether an operator is normal or not, and, if it is not normal, the degree of nonnormality, affect the convergence of iterative solvers such as GMRES.

An important question, therefore, is whether the operators $S, K, T$ and $A_{\eta}$ are normal on any domains other than the circle (in 2-d, or sphere in 3-d). Numerical experiments in Betcke \& Spence (2011) demonstrate that the combined potential operator $A_{\eta}$ appears to be nonnormal on a wide range of 2-d domains, which leads to the question of whether the circle is the only 2-d domain on which these operators are normal. In this paper we prove for the single layer potential operator $S$ that, amongst all sufficiently smooth 2-d and 3-d domains, balls are the only domains for which the operator is normal in the standard $L^{2}(\Gamma)$ inner product (see Theorem 3.1 below). The rest of this paper is, in some sense, an investigation into whether the desirable property of normality can be recovered, either in special cases or approximately.

If the Helmholtz equation is separable in a particular orthogonal coordinate system, then the Green's function can be expanded in terms of special functions ((1.2) is this expansion for polar coordinates), and this allows the derivation of eigenvalue decompositions of the operators $S, K$, and $T$ for certain domains. We show that these decompositions lead to the operators being normal in a modified $L^{2}$ inner product, and demonstrate this in the case on an ellipse.

The explicit eigenvalue decompositions above are only possible for a very restrictive class of domains. Nevertheless, for arbitrary smooth domains we derive an approximate eigenvalue decomposition that relates eigenvalues on smooth domains to eigenvalues on the unit circle. In particular, it allows us to separate the spectrum into $\mathscr{O}(k)$ eigenvalues that are not well approximated by circle eigenvalues, and a tail of eigenvalues that are well-approximated by circle eigenvalues with associated approximate eigenfunctions that are orthogonal in a modified $L^{2}$ inner product. This result shows that nonnormality appears mainly to affect a finite number of $\mathscr{O}(k)$ eigenvalues.

The paper is organized as follows. In Section 2 we briefly introduce two related tools for the study of nonnormality, namely pseudospectra and numerical ranges. We then demonstrate the effect of nonnormality on norm bounds and on coercivity constants for boundary integral operators in acoustic scattering. In Section 3 we present a proof that, amongst all sufficiently smooth domains, circles (respectively balls in 3-d) are the only ones for which the acoustic single layer potential is normal. The proof is based on transforming the question of normality into a uniqueness problem of equilibrium potentials, which has been solved by Reichel (1997). In Section 4 we use the decomposition of the acoustic Green's function in elliptic coordinates to derive an eigenvalue decomposition of the operators $S, K$ and $T$ on the boundary of an ellipse. This allows one to prove that these operators are normal in a scaled $L^{2}$ inner product. In Section 5 approximate eigendecompositions are derived for general analytic domains. Applications of the results to the combined potential operator $A_{\eta}$ are treated in Section 6. Numerical examples will be given for two 
domains. The paper finishes with conclusions in Section 7. An extended version of this paper with full proofs of the Theorems 4.1 and 5.1, and derivations of the asymptotic behavior of the eigenvalues of $S, K$, and $T$ can be found in Betcke et al. (2012).

Notation in this paper is mostly standard. Unless otherwise stated we use $\|\cdot\|$ for the norm induced by the standard complex $L^{2}(\Gamma)$ inner product

$$
(u, v):=\int_{\Gamma} u(y) \overline{v(y)} d s(y),
$$

where $\Gamma$ is the boundary of a given domain. For disambiguation we will also use from time to time the symbols $\|\cdot\|_{L^{2}(\Gamma)}$ and $(\cdot, \cdot)_{L^{2}(\Gamma)}$ to emphasise the standard $L^{2}(\Gamma)$ norm and inner product. Frequently we will make use of a scaled $L^{2}$ inner product defined by

$$
(u, v)_{L^{2}\left(F^{-1}, \Gamma\right)}:=\int_{\Gamma}\left(F^{-1} u\right)(y) \overline{v(y)} d s(y)
$$

and the induced norm

$$
\|v\|_{L^{2}\left(F^{-1}, \Gamma\right)}:=\left(\int_{\Gamma}\left(F^{-1} v\right)(y) \overline{v(y)} d s(y)\right)^{1 / 2},
$$

where $F: L^{2}(\Gamma) \rightarrow L^{2}(\Gamma)$ is a given positive multiplication operator defined as $[F v](x)=f(x) v(x)$ for a positive function $f \in L^{\infty}(\Gamma)$. Note that for the $L^{2}(\Gamma)$ norm of the operator $F$ it holds that $\|F\|_{L^{2}(\Gamma)}=$ $\|f\|_{L^{\infty}(\Gamma)}$. With $v \in L^{2}(\Gamma)$, the Cauchy-Schwarz inequality implies that

$$
\|v\|_{L^{2}\left(F^{-1}, \Gamma\right)} \leqslant\left\|F^{-1}\right\|_{L^{2}(\Gamma)}^{1 / 2} \cdot\|v\|_{L^{2}(\Gamma)} .
$$

The reverse inequality is obtained as

$$
\|v\|_{L^{2}(\Gamma)}=\left\|F^{1 / 2} F^{-1 / 2} v\right\|_{L^{2}(\Gamma)} \leqslant\|F\|_{L^{2}(\Gamma)}^{1 / 2}\|v\|_{L^{2}\left(F^{-1}, \Gamma\right)} .
$$

Combining (1.5) and (1.6) we have the following proposition.

PROPOSITION 1.2 Let $A: L^{2}(\Gamma) \rightarrow L^{2}(\Gamma)$ be a bounded linear operator. Then

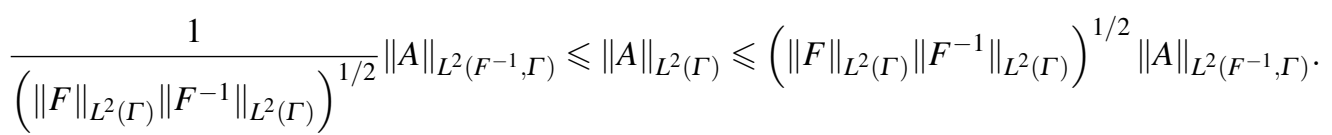

Proof. The proof follows from

$$
\frac{\|A v\|_{L^{2}\left(F^{-1}, \Gamma\right)}}{\|v\|_{L^{2}\left(F^{-1}, \Gamma\right)}} \leqslant\left(\|F\|_{L^{2}(\Gamma)}\left\|F^{-1}\right\|_{L^{2}(\Gamma)}\right)^{1 / 2} \frac{\|A v\|_{L^{2}(\Gamma)}}{\|v\|_{L^{2}(\Gamma)}}
$$

and

$$
\frac{\|A v\|_{L^{2}(\Gamma)}}{\|v\|_{L^{2}(\Gamma)}} \leqslant\left(\|F\|_{L^{2}(\Gamma)}\left\|F^{-1}\right\|_{L^{2}(\Gamma)}\right)^{1 / 2} \frac{\|A v\|_{L^{2}\left(F^{-1}, \Gamma\right)}}{\|v\|_{L^{2}\left(F^{-1}, \Gamma\right)}} .
$$

For the numerical experiments in this paper we use a simple self-developed Python based 2-d Galerkin boundary element code. The problems are discretised by choosing quadratic polynomials on each element as basis functions. The number of elements for each computation was refined until the results were stable up to plotting accuracy.

\section{Nonnormality and its consequences for the solution of boundary integral equations}

In this section we first give a brief introduction to two widely used tools for the analysis of nonnormality, namely pseudospectra and the numerical range. Then by looking at norms and coercivity constants we give examples of how nonnormality influences the behavior of boundary integral operators. 


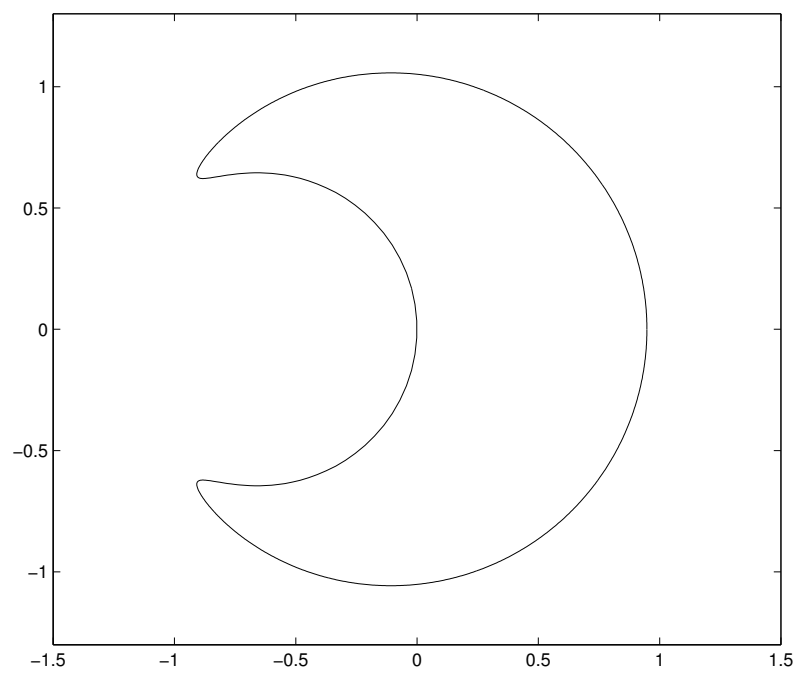

FIG. 1: A crescent domain parameterised by $z(t)=e^{i t}-\frac{0.1}{e^{i t}+0.9}, t \in[0,2 \pi]$.

\subsection{Pseudospectra and numerical ranges of operators}

A widely used tool for investigating nonnormality is via the pseudospectrum of an operator. It can be characterised by the following three equivalent definitions (Trefethen \& Embree, 2005).

DEFINITION 2.1 (Pseudospectrum of a bounded operator) Let $A$ be a bounded operator on a Hilbert space $\mathscr{H}$ and $\varepsilon>0$ be arbitrary: The $\varepsilon$-pseudospectrum $\sigma_{\varepsilon}(A)$ of $A$ is the set of $z \in \mathbb{C}$ defined equivalently by any of the conditions

1. $\left\|(z-A)^{-1}\right\|>\varepsilon^{-1}$.

2. $z \in \sigma(A+E)$ for some bounded operator $E$ with $\|E\|<\varepsilon$.

3. $z \in \sigma(A)$ or $\|(z-A) u\|<\varepsilon$ for some $u \in \mathscr{H}$ with $\|u\|=1$.

If $\|(z-A) u\|<\varepsilon$ with $\|u\|=1$ then $z$ is an $\varepsilon$-pseudoeigenvalue of $A$ and $u$ is a corresponding $\varepsilon$-pseudoeigenvector (or pseudoeigenfunction or pseudomode).

The definition in (Trefethen \& Embree, 2005, Chapter 4) is stated in terms of closed operators acting on Banach spaces. However, in this paper we are only interested in bounded Hilbert space operators. For normal matrices the $\varepsilon$-pseudospectrum is just the union of open disks of radius $\varepsilon$ around the spectral values. In general, if $A$ is nonnormal the form of the pseudospectra can be very different.

A rougher but also very useful tool to investigate nonnormality is the numerical range of a bounded linear operator $A$ acting on a Hilbert space $\mathscr{H}$. It is defined as

$$
W(A):=\{\langle A u, u\rangle, u \in \mathscr{H},\|u\|=1\} .
$$

The numerical range is always a convex set such that the spectrum $\sigma(A) \subset \overline{W(A)}$. Furthermore, the closure of the numerical range of a normal operator is the convex hull of its spectrum. There exists a simple relationship between the numerical range and the $\varepsilon$-pseudospectrum, namely

$$
\sigma_{\varepsilon}(A) \subset W(A)+\Delta_{\varepsilon}
$$

where $\Delta_{\varepsilon}$ is the open disk around the origin of radius $\varepsilon$ (Trefethen \& Embree, 2005, Chapter 17). In Figure 2 we plot the $\varepsilon$-pseudospectrum and the numerical range for the combined potential operator $A_{\eta}$ in $L^{2}(\Gamma)$ with $\eta=k=10$ for the crescent domain shown in Figure 1 (see Section 6 for more examples and details of the computation). The differently coloured contour lines are the boundaries of the $\varepsilon$-pseudospectrum for 


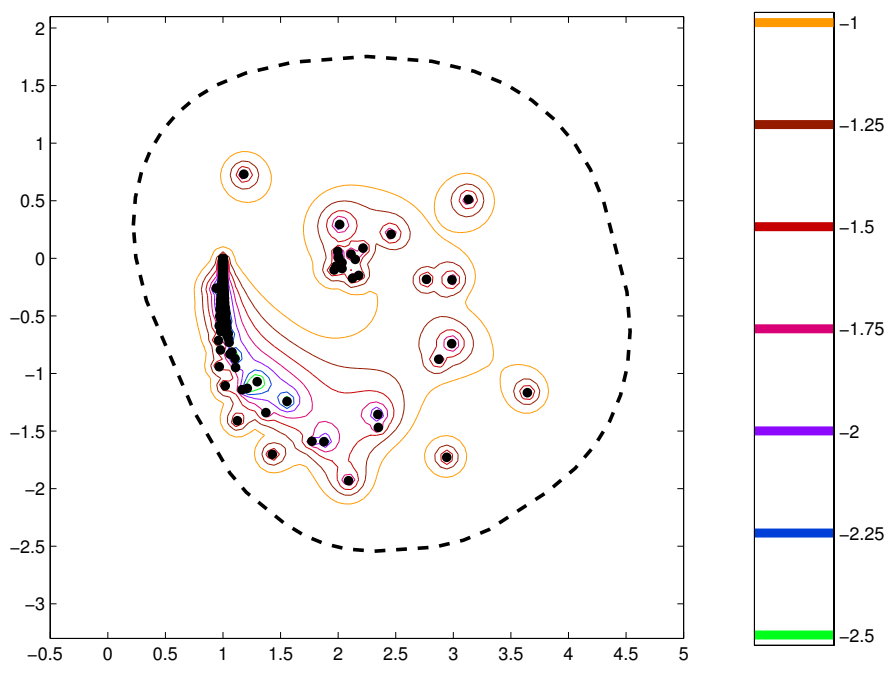

FIG. 2: Contour lines of the $\varepsilon$-pseudospectrum of the operator $A_{\eta}$ for $\eta=k=10$ on the crescent domain, where $\varepsilon=10^{\tau}$ with the values of $\tau$ given in the legend. The dashed line is the boundary of the numerical range $W\left(A_{\eta}\right)$.

$\varepsilon=10^{\tau}$, where $\tau$ are the values given in the legend. The dashed line is the boundary of the numerical range and the dots are the eigenvalues. It is clear from this plot that the operator is not normal. The numerical range is much larger than the convex hull of the eigenvalues. The $\varepsilon$-pseudospectrum was computed using EigTool, a Matlab software package developed by Wright (2002).

\subsection{Norm estimates}

If the compact operator $A$ is normal then by diagonalisation in its eigenbasis it follows that

$$
\|A\|=\sup _{n}\left|\lambda_{n}(A)\right| .
$$

This was used in Domínguez et al. (2007) to give estimates for the $k$-dependence of the norm of combined boundary integral operators on the circle in 2-d and the sphere in 3-d (following similar investigations by Kress \& Spassov (1983), Kress (1985)). From the analysis in (Domínguez et al., 2007, Section 4) it follows that

$$
\|S\| \leqslant C k^{-2 / 3},\|K\| \leqslant C k^{1 / 3}
$$

for the circle. Numerical experiments in Betcke et al. (2011) indicated that a sharper bound on $\|K\|$ is

$$
\|K\| \leqslant C .
$$

Indeed, this bound was proved for the unit sphere in 3-d in Banjai \& Sauter (2007), and essentially the same argument shows that this bound also holds for the unit circle (Chandler-Wilde et al., 2012, Theorem 5.12). In Figure 3 we show the $k$-dependence of the norm of the operators $S, K$ and $T$ on the unit circle. The results were computed by taking the maximum over the absolute values of the first 100 eigenvalues computed from the formulas in Theorem 1.1. These computations confirm both the $O\left(k^{-2 / 3}\right)$ decay for the single layer potential and the fact that the norms of $K$ and $T$ behave like $O(1)$ independently of $k$.

If $A$ is nonnormal we cannot use eigenvalues to bound the norm since in general we only have

$$
\sup _{n}\left|\lambda_{n}(A)\right| \leqslant\|A\|
$$

In Figure 4 we demonstrate the difference of $\left|\lambda_{\max }\right|:=\sup _{n}\left|\lambda_{n}\right|$ and $\|S\|_{L^{2}(\Gamma)}$ for the single layer potential 


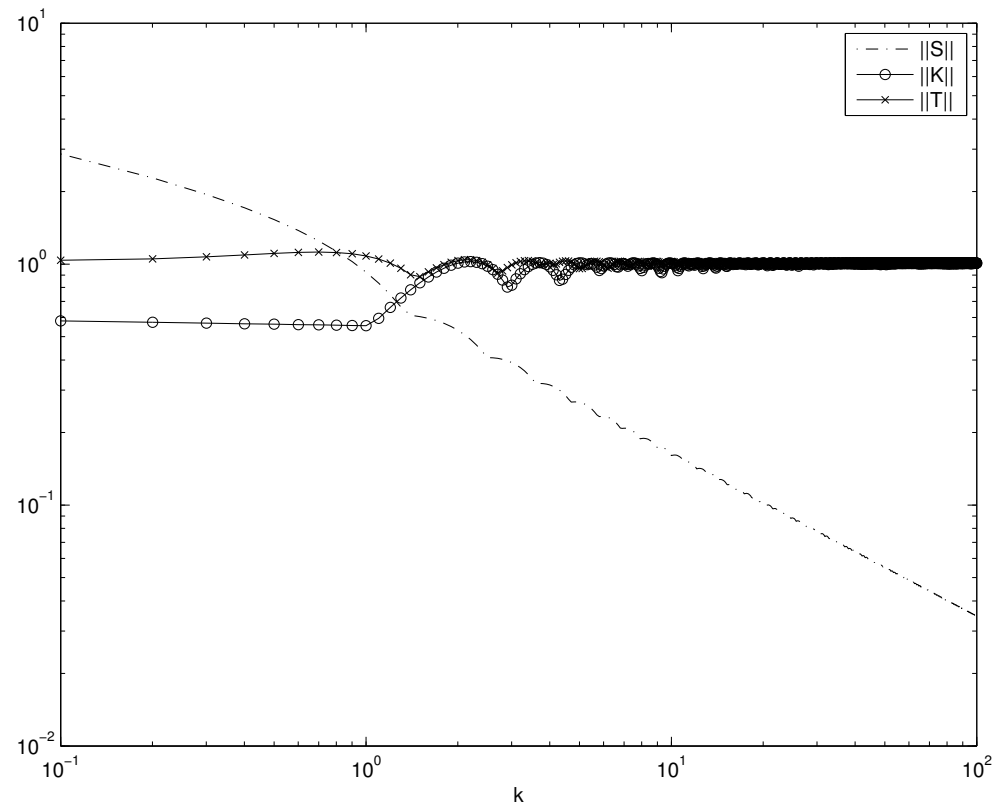

FIG. 3: The norm of the operators $\|S\|,\|K\|$ and $\|T\|$ on the unit circle.



FIG. 4: Absolute value of the largest eigenvalue $\lambda_{\max }(S)$ and $\|S\|_{L^{2}(\Gamma)}$ of the single layer potential $S$ on the boundary of the crescent domain from Figure 1 for growing values $k$. 
on the boundary $\Gamma$ for the crescent domain from Figure 1. Numerically, for the eigenvalues we obtain a decay of approximately $O\left(k^{-0.77}\right)$, while the norm decays much slower with a rate of about $O\left(k^{-1 / 2}\right)$. This effect is a consequence of nonnormality. In Betcke et al. (2011) various norm estimates are summarised; for general Lipschitz domains the estimate $\|S\|_{L^{2}(\Gamma)}=O\left(k^{-1 / 2}\right)$ holds.

There is an interesting relationship between the norm of an operator and its numerical range. Let $r(A):=\sup \{|z|: z \in W(A)\}$ be the numerical radius of $A$. Then it holds that $r(A) \leqslant\|A\| \leqslant 2 r(A)$ (Gustafson $\&$ Rao, 1997). Hence, $k$-dependent norm bounds immediately are also bounds for the extent of the numerical range of the operators $S, K$ and $T$.

\subsection{Coercivity Constants}

An operator $A$ acting on a Hilbert space $\mathscr{H}$ is coercive if there exists $\gamma>0$, such that

$$
\gamma\|u\|^{2} \leqslant|\langle A u, u\rangle|
$$

for all $u \in \mathscr{H}$. Consider the abstract variational problem

$$
\text { Find } u \in \mathscr{H} \text {, such that }\langle A u, v\rangle=f(v), \quad \forall v \in \mathscr{H},
$$

where $f$ is an element of the dual space $\mathscr{H}^{\prime}$. Let $\mathscr{V}^{(h)}$ be a finite dimensional subspace of $\mathscr{H}$ and denote by $u^{(h)}$ the solution of the variational problem (2.1) restricted to $\mathscr{V}^{(h)}$. Then if $A$ is coercive, by Céa's Lemma, (Cea, 1964), we have

$$
\left\|u-u^{(h)}\right\| \leqslant C \min _{v \in \mathscr{V}(h)}\left\|u^{(h)}-v\right\|
$$

where $C=\|A\| / \gamma$.

The analysis of coercivity constants for the combined potential operator $A_{\eta}$ is the subject of current research (see Spence et al. (2011)), and the main motivation for this is the following. Recall that establishing coercivity means that the quasi-optimality estimate (2.2) holds for any finite dimensional subspace $\mathscr{V}^{(h)}$. Recently much research effort has gone into designing $k$-dependent subspaces that accurately approximate the solution to scattering problems where $k$ is large, even for relatively small subspace dimensions. (Recall that the standard piecewise polynomial subspaces require their dimension to grow like $k^{d-1}$ to maintain accuracy as $k$ increases.) An overview of these "hybrid numerical-asymptotic" methods can be found in the review by Chandler-Wilde et al. (2012). Since these subspaces depend on $k$ in a complicated way, the only known way of proving convergence of Galerkin methods using these subspaces is via coercivity.

A first coercivity proof for $A_{k}$ in $L^{2}(\Gamma)$ was given in Domínguez et al. (2007) for the case that $\Gamma$ is the unit circle or the unit sphere. By a careful analysis of the eigenvalues of $A_{k}$ it was shown that $\gamma \geqslant 1$ for sufficiently large $k$. For general domains an eigenvalue analysis is not sufficient any more. This can be seen as follows. From the definition of the numerical range it follows that $A_{\eta}$ is coercive if and only if $0 \notin \overline{W\left(A_{\eta}\right)}$. Furthermore, if $A_{k}$ is coercive then $\gamma$ is just the distance of 0 to $\overline{W\left(A_{k}\right)}$. In the case of the circle, the combined potential operator is normal and the numerical range is just the convex hull of the eigenvalues, but this need not be the case for more general domains. However, we can still numerically study the distance of the origin to the boundary of the numerical range in order to estimate coercivity constants. This was recently done in Betcke \& Spence (2011). As an example consider the numerical range plot in Figure 2 for $A_{k}$ defined on the boundary of the crescent domain. From the plot it follows that $A_{k}$ is coercive with a coercivity constant smaller than 0.5 . It is instructive to compare the boundary of the numerical range with the eigenvalues. The boundary of $W\left(A_{k}\right)$ is much closer to 0 than the eigenvalues, an effect due to the nonnormaliity of the operator.

The currently best available result on the coercivity of $A_{k}$ is that it is coercive on $L^{2}(\Gamma)$, for $k$ large enough, with any coercivity constant less than one, when $\Omega$ is a smooth convex 2- or 3-d domain with strictly positive curvature (Spence et al., 2012). The numerical computations of Betcke \& Spence (2011) indicate that coercivity holds for a wide class of domains (at least in 2-d), but proving this remains open.

\section{A nonnormality proof for the single layer potential operator}

It was observed in (Betcke \& Spence, 2011, Lemma 3.5) that $A_{\eta}$ is normal in $L^{2}(\Gamma)$ if $\Gamma$ is the boundary of a circle in 2-d or a sphere in 3-d. Using the same argument $S, K$ and $T$ are also normal on the circle 
and sphere. In this section we provide a partial answer to the question of whether there are any domains, other than the circle and sphere, for which the operators $S, K, T$ and $A_{\eta}$ are normal in the standard $L^{2}(\Gamma)$ inner product. By reformulating the problem of normality into one of equilibrium potentials we prove that among all bounded $C^{2, \alpha}$ domains balls are the only ones for which the operator $S$ is normal. The main result is the following theorem.

THEOREM 3.1 Let $\Omega \subset \mathbb{R}^{d}, d=2,3$ be a bounded $C^{2, \alpha}$ domain with boundary $\Gamma$. Then the single layer potential $S$ is normal if and only if $\Omega$ is a ball.

The proof that $S$ is normal on a ball follows by diagonalisation in the Fourier-basis (2-d) and by diagonalisation in spherical harmonics (3-d), see (Betcke \& Spence, 2011, Lemma 3.5) for details. To prove the converse we first show that $S$ normal implies that the logarithmic single layer potential operator defined by

$$
\left[\mathscr{S}_{0} \phi\right](x):=\left\{\begin{array}{cc}
-\frac{1}{2 \pi} \int_{\Gamma} \log |x-y| \phi(y) d s(y), & 2-\mathrm{d} \\
\frac{1}{4 \pi} \int_{\Gamma} \frac{1}{|x-y|} \phi(y) d s(y), & 3-\mathrm{d}
\end{array}\right.
$$

applied to the constant function 1 is constant inside $\Omega$. We can then use the following result to show that $\Omega$ must be a ball.

THEOREM 3.2 (Theorem 2 and $\S 3$ of Reichel (1997)) If $\Omega$ is a $C^{2, \alpha}$ domain and $\mathscr{S}_{0} 1$ is constant in $\Omega$ then $\Omega$ is a ball.

This problem can be understood in terms of equilibrium potentials (see, e.g., (Ransford, 1995, §3.3)). Given a domain, the nontrivial measure that gives rise to a constant potential throughout the domain is called the equilibrium measure. The corresponding potential is the equilibrium potential. Theorem 3.2 states that the potential $\mathscr{S}_{0} 1$ is the equilibrium potential only for the case when $\Omega$ is a ball. The proof in Reichel (1997) considers the equivalent problem of showing that the following overdetermined boundary value problem has a solution if and only if $\Omega$ is a ball:

$$
\Delta w=0 \quad \text { in } \Omega, \quad \gamma_{1}^{+} w=-1, \quad \gamma_{0}^{+} w=a,
$$

where $a$ is a constant and an appropriate condition at infinity is prescribed. This condition at infinity is $u \rightarrow 0$ for $d \geqslant 3$, and one involving layer potentials for $d=2$ (since the single layer potential does not decay at infinity in this case).

These overdetermined problems in potential theory have a long history, beginning with the celebrated work of Serrin (1971), and are motivated by physical questions including stress in hydrodynamics.

A natural question is where the restriction that $\Omega$ is a $C^{2, \alpha}$ domain comes from. In order to apply the appropriate theorem on the overdetermined problem, (Reichel, 1997, Theorem 1), Reichel requires that $\mathscr{S}_{0} 1$ be in $C^{2}\left(\overline{\Omega^{+}}\right)$. Reichel uses a special case of a general regularity theorem (Gilbarg \& Trudinger, 1998, Theorem 6.14) to show that if $D$ is a $C^{2, \alpha}$ domain, $\Delta u=0$ in $D$, and the boundary data is $C^{2, \alpha}$, then $u \in C^{2, \alpha}(\bar{D})$.

In order to prove Theorem 3.1 from Theorem 3.2 we need to show that if the acoustic single layer potential $S$ is normal then $\mathscr{S}_{0} 1$ is constant in $\Omega$. The next lemma is technical and gives a reformulation of the condition that $S$ is normal.

LEMMA 3.1 Let the acoustic Green's function $g(x, y)=u(|x-y|)+i v(|x-y|)$ where $u$ and $v$ are real. $S$ is normal if and only if

$$
C(x, z):=\int_{\Gamma} u(|z-y|) v(|x-y|)-u(|x-y|) v(|z-y|) d s(y)=0
$$

for all $x, z \in \Gamma$.

Proof. Given $f \in L^{2}(\Gamma)$

$$
\begin{aligned}
{\left[\left(S S^{*}-S^{*} S\right) f\right](x) } & =\int_{\Gamma} \int_{\Gamma}(g(x, y) \bar{g}(z, y)-\bar{g}(y, x) g(y, z)) f(z) d s(z) d s(y) \\
& =\int_{\Gamma} \int_{\Gamma} 2 i(u(|z-y|) v(|x-y|)-u(|x-y|) v(|z-y|)) f(z) d s(z) d s(y) \\
& =2 i \int_{\Gamma} f(z) C(x, z) d s(z)
\end{aligned}
$$

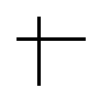


where $x, z \in \Gamma$. Interchanging the order of integration is justified by Fubini's theorem since $g$ is weakly singular.

Assume that $S$ is normal, that is $S S^{*}-S^{*} S=0$. Given any arbitrary $z^{\prime} \in \Gamma$, the right hand side of (3.2) can be made arbitrarily close to $2 i C\left(x, z^{\prime}\right)$ by choosing an appropriate $f(z)$ with support in a neighbourhood of $z^{\prime}$. Thus if $\left(S S^{*}-S^{*} S\right)(f)(x)$ is equal to zero for all $x \in \Gamma$ and for all $f$ then $C\left(x, z^{\prime}\right)=0$ for all $x, z^{\prime} \in \Gamma$. The converse is immediate. $\quad$

We know that $S$ is normal for $\Gamma$ the circle or sphere, and we can check that $C(x, z)=0$ in this case. Indeed, the change of variables $y=(x+z)-y^{\prime}$ transforms the unit circle/sphere centered at 0 to one centred at $x+z$, and $d s(y)=d s\left(y^{\prime}\right)$. Making this change of variables in the second term in (3.1) we see that, since $|z-y|=\left|x-y^{\prime}\right|$ and $|x-y|=\left|z-y^{\prime}\right|$, the second term cancels with the first and thus $C(x, z)=0$.

For the next lemma we note that for $d=2, u(r)=-\frac{1}{4} Y_{0}(k r)$ and $v(r)=\frac{1}{4} J_{0}(k r)$, where $J_{v}$ and $Y_{v}$ are Bessel functions of the first and second kind respectively. For $d=3, g(x, y)=e^{i k|x-y|} /(4 \pi|x-y|)$ and thus $u(r)=(\cos k r) /(4 \pi r)$ and $v(r)=(\sin k r) /(4 \pi r)$.

We can now prove the following.

LEMmA 3.2 If $C(x, z)=0$ for all $x, z \in \Gamma$ then $\mathscr{S}_{0} 1$ is constant in $\Omega$.

Proof. Since $C(x, z)=0$ for all $x, z \in \Gamma$, then of course for every fixed $z C(x, z)=0$ for all $x \in \Gamma$. Thus, $\nabla_{\Gamma, x} C(x, z)=0$ for all $x \in \Gamma$, where $\nabla_{\Gamma, x}$ is the surface gradient. An explicit expression for this in terms of a parametrisation of the boundary can be found in (Monk, 2003, §3.4) (Colton \& Kress, 1983, §2.1) for smooth domains and (Chandler-Wilde et al., 2012, Equation (A.14)) for Lipschitz domains. Furthermore, if $w$ is $C^{1}$ in a neighbourhood of $\Gamma$ then

$$
\nabla_{\Gamma, x} w(x)=\nabla w(x)-n(x) \frac{\partial w}{\partial n}(x), \quad x \in \Gamma .
$$

Ideally we would like to take $\nabla_{\Gamma, x}$ under the integral sign in the definition of $C(x, z)$ in (3.1) and obtain

$$
\nabla_{\Gamma, x} C(x, z)=P . V . \int_{\Gamma}\left(\frac{x-y}{|x-y|}-\frac{(x-y) \cdot n(x)}{|x-y|} n(x)\right)\left(u(|z-y|) v^{\prime}(|x-y|)-u^{\prime}(|x-y|) v(|z-y|)\right) d s(y)
$$

where we are using (3.3) to find the surface gradient of $|x-y|$ under the integral sign. However, since the integrand is weakly singular, this interchange of integration and differentiation requires some justification.

The key result is that, for $\phi \in L^{2}(\Gamma)$ and $k \geqslant 0$,

$$
\nabla_{\Gamma, x}[S \phi](x)=\left[\nabla_{\Gamma} S \phi\right](x)
$$

where the right hand side is the integral operator defined by

$$
\begin{aligned}
{\left[\nabla_{\Gamma} S \phi\right](x) } & =P . V . \int_{\Gamma} \nabla_{\Gamma, x} g(x, y) \phi(y) d s(y), \\
& =P . V . \int_{\Gamma}\left(\frac{x-y}{|x-y|}-\frac{(x-y) \cdot n(x)}{|x-y|} n(x)\right)\left(u^{\prime}(|x-y|)+i v^{\prime}(|x-y|)\right) \phi(y) d s(y) .
\end{aligned}
$$

On $C^{2}$ domains this follows from the results for Hölder continuous $\phi$ in (Colton \& Kress, 1983, Theorem 2.17) (using the density of Hölder continuous functions in $L^{2}(\Gamma)$ when $\Gamma$ is $C^{2}$ ), and this result is true even on Lipschitz domains by the harmonic analysis results surveyed in (Meyer \& Coifman, 2000, Chapter 15).

The result (3.5) justifies (3.4) as follows. $C$, defined by (3.1), consists of two terms: the first, viewed as a function of $x$, is the imaginary part of the single layer potential with density $u(|z-y|)$, the second, again viewed as a function of $x$, is the real part of the single layer potential with density $v(|z-y|)$. Both $u(|z-y|)$ and $v(|z-y|)$ are in $L^{2}(\Gamma)$ and thus interchanging the differentiation and integration is justified by the imaginary and real parts respectively of (3.5).

Since $\nabla_{\Gamma, x} C(x, z)=0$ for all $x \in \Gamma$ in particular this holds for $x=z$. Let $\chi(z):=\left.\nabla_{\Gamma, x} C(x, z)\right|_{(x=z)}$. Then, for $d=2$ the Wronskian $u(r) v^{\prime}(r)-u^{\prime}(r) v(r)=-1 /(2 \pi k r)$ and

$$
\chi(z)=P . V . \int_{\Gamma}\left(\frac{z-y}{|z-y|}-\frac{(z-y) \cdot n(z)}{|z-y|} n(z)\right) \frac{-1}{2 \pi k|z-y|} d s(y)=-\frac{1}{2 \pi k} \nabla_{\Gamma, z} \int_{\Gamma} \log (|z-y|) d s(y), \quad d=2,
$$


(where taking the surface gradient out from under the integral sign is justified by (3.5) above with $k=0$ ). For $d=3$, the Wronskian $u(r) v^{\prime}(r)-u^{\prime}(r) v(r)=k /\left(4 \pi r^{2}\right)$ and

$$
\chi(z)=P . V . \int_{\Gamma}\left(\frac{z-y}{|z-y|}-\frac{(z-y) \cdot n(z)}{|z-y|} n(z)\right) \frac{k}{4 \pi|z-y|^{2}} d s(y)=\frac{k}{4 \pi} \nabla_{\Gamma, z} \int_{\Gamma} \frac{1}{|z-y|} d s(y), \quad d=3 .
$$

Thus $C(x, z)=0$ for all $x, z \in \Gamma$ implies that $\chi(z)=0$ for all $z \in \Gamma$ and thus $\left[\mathscr{S}_{0} 1\right](z)$ is constant for all $z \in \Gamma$. Since $\mathscr{S}_{0} 1$ is harmonic, by the maximum principle it is constant in $\Omega$.

By combining Lemma 3.1 and Lemma 3.2 we can apply Theorem 3.2 to show that if $S$ is normal then $\Omega$ must be a ball. This concludes the missing direction of the proof of Theorem 3.1.

\section{The spectrum on the boundary of an ellipse}

\subsection{Eigendecomposition in elliptic coordinates}

The main ingredient for computing an eigenvalue expansion on the disk is the Graf addition formula that gives an expansion of the acoustic Green's function in a polar coordinate system. In this section we generalise these results to an ellipse by using an elliptic coordinate system and an expansion of the acoustic Green's function in elliptic coordinates. A significant difference to the disk case is that the natural parameterisation of the boundary of an ellipse in elliptic coordinates has a non-constant Jacobian. The effect is that instead of a simple eigenvalue expansion for the single layer potential $S$ we will arrive at a generalised eigenvalue expansion of the form $S F^{-1} u_{n}=\lambda_{n} u_{n}$, where $F$ is a simple scaling operator. For the case of boundary integral operators in harmonic potential theory eigenvalue expansions on the ellipse were derived by Rodin \& Steinbach (2003). For the Helmholtz case we need Mathieu functions, which we will briefly introduce here.

Let $\Omega$ be an ellipse with boundary $\Gamma$ parameterised by $\gamma(v):=\left[\begin{array}{c}a \cosh \mu \cos v \\ a \sinh \mu \sin v\end{array}\right], v \in[0,2 \pi]$. Define $q=\frac{1}{4}(k a)^{2}$. Separation of variables of the Helmholtz equation in the elliptic variables $(\mu, v)$ leads to the standard Mathieu equation

$$
N^{\prime \prime}(v)+(\lambda-2 q \cos 2 v) N(v)=0
$$

and the modified Mathieu equation

$$
M^{\prime \prime}(\mu)-(\lambda-2 q \cosh 2 \mu) M(\mu)=0,
$$

where $\lambda$ is a separation constant. Together with suitable boundary conditions (4.1) is a Sturm-Liouville problem, where $\lambda=\lambda(q)$ is the eigenvalue parameter. Depending on the boundary conditions two sets of eigenvalues $a_{n}(q)$ and $b_{n}(q)$ are defined (NIST Digital Library, $\S 28.2(\mathrm{v})$ ). By $c e_{n}(v, q)$ we denote the even eigenfunctions associated with the eigenvalues $a_{n}(q)$ and by $s_{n}(v, q)$ the odd eigenfunctions associated with $b_{n}(q)$. (NIST Digital Library, $\S 28.2(v i)$ ). These form an orthogonal basis of $L^{2}[0,2 \pi]$. For the normalisation of the functions we use the standard choice

$$
\int_{0}^{2 \pi} c e_{n}^{2}(v, q) d v=\int_{0}^{2 \pi} s e_{n}^{2}(v, q) d v=\pi
$$

We now consider the modified Mathieu equation (4.2) with the separation constant $\lambda$ equal to either $a_{n}(q)$ or $b_{n}(q)$ (i.e. the eigenvalues of (4.1)). With $\lambda=a_{n}(q)$ we introduce two sets of solutions to (4.2), $M c_{n}^{(1)}(\mu, q)$ and $M c_{n}^{(2)}(\mu, q)$, defined by their large $\mu$ asymptotics to be the elliptic analogues of the Bessel functions $J_{n}$ and $Y_{n}$ respectively (NIST Digital Library, eq. 28.20.11-12). From these the modified Mathieu functions $M c_{n}^{(3 / 4)}$ are defined by

$$
M c_{n}^{(3 / 4)}(\mu, q)=M c_{n}^{(1)}(\mu, q) \pm i M c_{n}^{(2)}(\mu, q) .
$$

It then holds that

$$
M c_{n}^{(3 / 4)}(\mu, q) \sim H_{n}^{(1 / 2)}\left(2 q^{1 / 2} \cosh \mu\right) \text { as } \mu \rightarrow \infty
$$

(NIST Digital Library, eq. 28.20.10,28.20.15-16), so that $M c_{n}^{(3 / 4)}$ is the analogue of $H_{n}^{(1 / 2)}$. One defines the functions $M s_{n}^{(1 / 2)}$ and $M s_{n}^{(3 / 4)}$ associated with the eigenvalues $b_{n}(q)$ in a similar way. 
Just as the acoustic Green's function can be expressed in terms of functions of polar coordinates (1.2), it can also be expanded in terms of functions of elliptic coordinates.

THEOREM 4.1 (Expansion of fundamental solution in Mathieu functions) The acoustic Green's function $\frac{i}{4} H_{0}^{(1)}(k|x-y|)$ can be expanded in terms of Mathieu functions as

$$
\begin{aligned}
\frac{i}{4} H_{0}^{(1)}(k|x-y|)=\frac{i}{2}( & \sum_{m=0}^{\infty} c e_{m}(v, q) c e_{m}\left(v^{\prime}, q\right) M c_{m}^{(1)}\left(\mu_{<}, q\right) M c_{m}^{(3)}\left(\mu_{>}, q\right) \\
& \left.+\sum_{m=1}^{\infty} s e_{m}(v, q) s e_{m}\left(v^{\prime}, q\right) M s_{m}^{(1)}\left(\mu_{<}, q\right) M s_{m}^{(3)}\left(\mu_{>}, q\right)\right),
\end{aligned}
$$

where $x=(a \cosh \mu \cos v, a \sinh \mu \sin v), y=\left(a \cosh \mu^{\prime} \cos v^{\prime}, a \sinh \mu^{\prime} \sin v^{\prime}\right)$ and $\mu_{>}=\max \left(\mu, \mu^{\prime}\right), \mu_{<}=$ $\min \left(\mu, \mu^{\prime}\right)$.

Proof. This formula can be found in (Morse \& Feshbach, 1953a, §11.2). However, it is not derived directly, but is quoted as a special case of a general technique described in (Morse \& Feshbach, 1953b, §7.2). Furthermore, the notation for Mathieu functions used in these references is not the (now fairly standard) notation used in the Digital Library of Mathematical Functions that we use here. Because of these complications we give a full derivation of this expansion in (Betcke et al., 2012, Appendix B).

We cannot quite proceed as in the case of the unit circle. On the ellipse the boundary measure $d s$ is not identical to the angular measure $d v$ since $\left|\gamma^{\prime}(v)\right| \neq$ const. We have to introduce an additional scaling operator $F$ to take this into account.

THEOREM 4.2 Let $\Gamma$ be the boundary of an ellipse and $x=\gamma(v)$ be its parameterisation in elliptic coordinates as defined above. Denote by $\mu$ the radius of the ellipse in elliptic coordinates associated with the parameter $q=\frac{1}{4}(k a)^{2}$. Define the multiplication operator $F: L^{2}(\Gamma) \rightarrow L^{2}(\Gamma)$ by $[F \phi](x)=\left|\gamma^{\prime}\left(\gamma^{-1}(x)\right)\right| \phi(x)$. The following eigenvalue decompositions hold.

- For the single layer potential $S$ :

$$
\begin{aligned}
S F^{-1} \phi_{j}^{(c)} & =\lambda_{j}^{(S, c)} \phi_{j}^{(c)} \\
S F^{-1} \phi_{j}^{(s)} & =\lambda_{j}^{(S, s)} \phi_{j}^{(s)},
\end{aligned}
$$

where

$$
\lambda_{j}^{(S, c)}=\frac{i \pi}{2} M c_{j}^{(1)}(\mu, q) M c_{j}^{(3)}(\mu, q), \quad \lambda_{j}^{(S, s)}=\frac{i \pi}{2} M s_{j}^{(1)}(\mu, q) M s_{j}^{(3)}(\mu, q)
$$

- For the double layer potential $K$ :

$$
\begin{aligned}
K \phi_{j}^{(c)} & =\lambda_{j}^{(K, c)} \phi_{j}^{(c)} \\
K \phi_{j}^{(s)} & =\lambda_{j}^{(K, s)} \phi_{j}^{(s)},
\end{aligned}
$$

where

$$
\lambda_{j}^{(K, c)}=\frac{i \pi}{2}\left[\frac{\partial}{\partial \mu} M c_{j}^{(1)}(\mu, q)\right] M c_{j}^{(3)}(\mu, q), \quad \lambda_{j}^{(K, s)}=\frac{i \pi}{2}\left[\frac{\partial}{\partial \mu} M s_{j}^{(1)}(\mu, q)\right] M s_{j}^{(3)}(\mu, q) .
$$

- For the conjugate double layer potential $T$ :

$$
\begin{aligned}
F T F^{-1} \phi_{j}^{(c)} & =\lambda_{j}^{(T, c)} \phi_{j}^{(c)} \\
F T F^{-1} \phi_{j}^{(s)} & =\lambda_{j}^{(T, s)} \phi_{j}^{(s)},
\end{aligned}
$$

where

$$
\lambda_{j}^{(T, c)}=\frac{i \pi}{2} M c_{j}^{(1)}(\mu, q)\left[\frac{\partial}{\partial \mu} M c_{j}^{(3)}(\mu, q)\right], \quad \lambda_{j}^{(T, s)}=\frac{i \pi}{2} M s_{j}^{(1)}(\mu, q)\left[\frac{\partial}{\partial \mu} M s_{j}^{(3)}(\mu, q)\right] .
$$


In all three cases the eigenfunctions $\phi_{j}^{(c)}$ and $\phi_{j}^{(s)}$ are defined as

$$
\phi_{j}^{(c)}(x)=c e_{j}\left(\gamma^{-1}(x), q\right), \quad \phi_{j}^{(s)}(x)=s e_{j}\left(\gamma^{-1}(x), q\right) .
$$

Proof. From the decomposition of the acoustic Green's function in Theorem 4.1 and the orthogonality of Mathieu functions with respect to the inner product $(\cdot, \cdot)_{L^{2}([0,2 \pi])}$ it follows for $x=(a \cosh \mu \cos v, a \sinh \mu \sin v)$ that

$$
\begin{aligned}
{\left[S F^{-1} \phi_{j}^{(c)}\right](x) } & =\int_{\Gamma} \frac{i}{4} H_{0}^{(1)}(k|x-y|) \frac{\phi_{j}^{(c)}(y)}{\left|\gamma^{\prime}\left(\gamma^{-1}(y)\right)\right|} d s(y) \\
& =\int_{0}^{2 \pi}\left|\gamma^{\prime}(t)\right| \frac{i}{4} H_{0}^{(1)}(k|x-\gamma(t)|) \frac{c e_{j}(t, q)}{\left|\gamma^{\prime}(t)\right|} d t \\
& =\frac{i \pi}{2} M c_{j}^{(1)}(\mu, q) M c_{j}^{(3)}(\mu, q) c e_{j}(v, q) \\
& =\lambda_{j}^{(S, c)} \phi_{j}^{(c)}(x) .
\end{aligned}
$$

For the double layer and conjugate double layer potential we first note that $\frac{\partial}{\partial n(x)}=\frac{1}{\left|\gamma^{\prime}\left(\gamma^{-1}(x)\right)\right|} \frac{\partial}{\partial \mu}$ for $x \in \Gamma$ since elliptic coordinates are an orthogonal coordinate system. Let $\tilde{x} \in \Omega^{+}$have the representation $(\tilde{\mu}, v)$ in elliptic coordinates. It follows that

$$
\begin{aligned}
{\left[\mathscr{K} \phi_{j}^{(c)}\right](\tilde{x}) } & =\int_{\Gamma} \frac{\partial}{\partial n(y)} \frac{i}{4} H_{0}^{(1)}(k|\tilde{x}-y|) c e_{j}\left(\gamma^{-1}(y), q\right) d s(y) \\
& =\frac{i \pi}{2}\left[\frac{\partial}{\partial \mu} M c_{j}^{(1)}(\mu, q)\right] M c_{j}^{(3)}(\tilde{\mu}, q) c e_{j}(v, q) .
\end{aligned}
$$

Since $K u=\gamma_{0}^{+} \mathscr{K}$ for $u \in L^{2}(\Gamma)$ the result follows from taking the limit $\tilde{\mu} \rightarrow \mu$. For the conjugate double layer potential we note that

$$
\left[\mathscr{S} F^{-1} \phi_{j}^{(c)}\right](\tilde{x})=\frac{i \pi}{2} M c_{j}^{(1)}(\mu, q) M c_{j}^{(3)}(\tilde{\mu}, q) c e_{j}(v, q)
$$

giving

$$
\left[F T F^{-1} \phi_{j}^{(c)}\right](x)=\left[F \gamma_{1}^{+} \mathscr{S} F^{-1} \phi_{j}^{(c)}\right](x)=\frac{i \pi}{2} M c_{j}^{(1)}(\mu, q)\left[\frac{\partial}{\partial \mu} M c_{j}^{(3)}(\mu, q)\right] c e_{j}(v, q)
$$

for $x \in \Gamma$. The calculations for $\phi_{j}^{(s)}$ are similar.

The operator $F$ is positive definite with bounded inverse on $L^{2}(\Gamma)$. We can therefore define the inner product and associated norm as in (1.3) and (1.4) respectively. Since $a \sinh \mu \leqslant\left|\gamma^{\prime}(t)\right| \leqslant a \cosh \mu$ for all $t \in \mathbb{R}$ it follows that

$$
\frac{1}{\sqrt{a \cosh \mu}}\|\cdot\|_{L^{2}(\Gamma)} \leqslant\|\cdot\|_{L^{2}\left(F^{-1}, \Gamma\right)} \leqslant \frac{1}{\sqrt{a \sinh \mu}}\|\cdot\|_{L^{2}(\Gamma)}
$$

(see also (1.5) and (1.6)).

We can now prove a simple normality result with respect to this scaled inner product.

THEOREM 4.3 The operators $S F^{-1}, K$ and $F T F^{-1}$ are normal with respect to the inner product $(u, v)_{L^{2}\left(F^{-1}, \Gamma\right)}$.

Proof. For any two functions $u, v \in L^{2}(\Gamma)$ we have

$$
(u, v)_{L^{2}\left(F^{-1}, \Gamma\right)}=\int_{\Gamma}\left(F^{-1} u\right) \bar{v} d s=\int_{0}^{2 \pi} u(\gamma(t)) \overline{v(\gamma(t))} d t .
$$

Hence, from the orthogonality of the Mathieu functions $c e_{j}$ and $s e_{j}$ with respect to the standard $L^{2}([0,2 \pi])$ inner product it follows that the functions $\phi_{j}^{(c)}$ and $\phi_{j}^{(s)}$ are orthogonal with respect to the inner product 
$(\cdot, \cdot)_{L^{2}\left(F^{-1}, \Gamma\right)}$. Together with Theorem 4.2 it therefore follows that the operators $S F^{-1}, K$ and $F T F^{-1}$ diagonalize in a unitary basis in $L^{2}\left(F^{-1}, \Gamma\right)$ proving normality.

For the single layer potential a particularly nice relationship follows immediately.

COROLlary 4.1 It holds that $S F^{-1} S^{*}=S^{*} F^{-1} S$.

Proof. The adjoint $\tilde{S}^{*}$ of $\tilde{S}:=S F^{-1}$ in $L^{2}\left(F^{-1}, \Gamma\right)$ is given by $\tilde{S}^{*} F:=S^{*} F^{-1}$ since

$$
(\tilde{S} f, g)_{L^{2}\left(F^{-1}, \Gamma\right)}=\left(F^{-1} S F^{-1} f, g\right)=\left(F^{-1} f, S^{*} F^{-1} g\right)=\left(f, \tilde{S}^{*} g\right)_{L^{2}\left(F^{-1}, \Gamma\right)}
$$

for all $f, g \in L^{2}\left(F^{-1}, \Gamma\right)$. From the normality of $\tilde{S}$ in $L^{2}\left(F^{-1}, \Gamma\right)$ it follows that $\tilde{S}$ commutes with its adjoint $\tilde{S}^{* F}$. Hence, $\tilde{S} \tilde{S}^{* F}=\tilde{S}^{* F} \tilde{S}$ and therefore

$$
S F^{-1} S^{*} F^{-1}=S^{*} F^{-1} S F^{-1},
$$

from which the result follows by multiplying from the right with $F$.

With $\tilde{\phi}_{j}^{(\tau)}:=F^{-1} \phi_{j}^{(\tau)}, \tau=c, s$ we can equivalently formulate the result of Theorem 4.2 as

$$
S \tilde{\phi}_{j}^{(\tau)}=\lambda_{j}^{(S, \tau)} F \tilde{\phi}_{j}^{(\tau)}, K \phi_{j}^{(\tau)}=\lambda_{j}^{(K, \tau)} \phi_{j}^{(\tau)}, T \tilde{\phi}_{j}^{(\tau)}=\lambda_{j}^{(T, \tau)} \tilde{\phi}_{j}^{(\tau)} .
$$

Hence, the single layer potential $S$ admits a generalized eigenvalue decomposition with eigenfunctions $\tilde{\phi}_{j}^{(\tau)}$ that are orthogonal in the $L^{2}(F, \Gamma)$ inner product. The operator $K$ admits a standard eigenvalue decomposition with eigenfunctions that are orthogonal in $L^{2}\left(F^{-1}, \Gamma\right)$ and $T$ admits a standard eigenvalue decomposition with eigenfunctions that are orthogonal in $L^{2}(F, \Gamma)$. It follows immediately that $K$ and $T$ are normal operators in $L^{2}\left(F^{-1}, \Gamma\right)$ and $L^{2}(F, \Gamma)$, respectively while the generalized pencil $(S, F)$ has the same spectrum as the normal operator $S F^{-1}$ in $L^{2}\left(F^{-1}, \Gamma\right)$.

Finally, normality in the scaled inner product can be used to obtain information about the operators in the usual $L^{2}(\Gamma)$ inner product. The starting point is the well-known bound on the resolvent of a bounded, normal operator $A$ on a Hilbert space in terms of its spectrum $\sigma(A)$ :

$$
\left\|(z I-A)^{-1}\right\|=\sup _{\lambda \in \sigma(A)} \frac{1}{|z-\lambda|},
$$

see, e.g., (Kato, 1995, eq. V.3.3.1). Together with Theorem 4.3 we can then derive the following resolvent estimates.

COROLlary 4.2 For the resolvents of $K$ and $T$ on an ellipse the following estimates hold. ${ }^{1}$

$$
\begin{aligned}
& \left\|(z I-K)^{-1}\right\|_{L^{2}(\Gamma)} \leqslant\left(\|F\|_{L^{2}(\Gamma)}\left\|F^{-1}\right\|_{L^{2}(\Gamma)}\right)^{1 / 2} \sup _{\lambda \in \sigma(K)} \frac{1}{|z-\lambda|} \\
& \left\|(z I-T)^{-1}\right\|_{L^{2}(\Gamma)} \leqslant\left(\|F\|_{L^{2}(\Gamma)}\left\|F^{-1}\right\|_{L^{2}(\Gamma)}\right)^{1 / 2} \sup _{\lambda \in \sigma(T)} \frac{1}{|z-\lambda|}
\end{aligned}
$$

Proof. From Proposition 1.2 we have that

$$
\left\|(z I-K)^{-1}\right\|_{L^{2}(\Gamma)} \leqslant\left(\|F\|_{L^{2}(\Gamma)}\left\|F^{-1}\right\|_{L^{2}(\Gamma)}\right)^{1 / 2}\left\|(z I-K)^{-1}\right\|_{L^{2}\left(F^{-1}, \Gamma\right)},
$$

and the result for $K$ now follows from the normality of $K$ in $L^{2}\left(F^{-1}, \Gamma\right)$ and (4.6). The result for $T$ follows similarly from the normality of this operator in $L^{2}(F, \Gamma)$ by exchanging the roles of $F$ and $F^{-1}$ in the above argument.

\footnotetext{
${ }^{1}$ We would like to thank one anonymous referee for pointing out this result to us.
} 


\subsection{A numerical example}

To illustrate the results of this section we compare the spectra and numerical ranges of the operators $S$, $K$ and $T$ in the standard $L^{2}(\Gamma)$ inner product on the ellipse with the spectra and numerical ranges of the operators $S F^{-1}, K$ and $F T F^{-1}$ in the $L^{2}\left(F^{-1}, \Gamma\right)$ inner product. The example domain is an ellipse with boundary defined by the parameters $a=1$ and $\mu=0.3$ in elliptic coordinates. The wavenumber is $k=10$. The results are presented in Figure 5. It is clearly visible that the standard operators $S, K$ and $T$ are not normal in $L^{2}(\Gamma)$ since the numerical ranges are not the closed convex hulls of the spectra of the operators (left plots in Figure 5). However, after scaling and changing the inner product up to the accuracy of the plotting scale the numerical ranges are the closed convex hulls of the spectra of $S F^{-1}, K$ and $F T F^{-1}$ (right plots in Figure 5). Note that the eigenvalues of $S F^{-1}$ are certainly not identical to those of $S$, while for $K$ and $T$ the rescaling from Theorem 4.2 does not change the eigenvalues.

\section{General smooth domains}

Since the technique used for the eigenvalue decomposition of the previous section (relying on the decomposition of the Green's function in an orthogonal coordinate system) is only applicable to a very restrictive class of domains, we derive in this section approximate decompositions for general smooth domains. The idea is to use the well-known result that the boundary integral operators $S, K$ and $T$ on the boundary of a sufficiently smooth domain can be represented as compact perturbations of the corresponding operators on the unit circle (Sloan, 1992). For the purpose of this section we will identify $\mathbb{R}^{2}$ with the complex plane $\mathbb{C}$. We also note that all the analysis of this section is for a fixed wavenumber $k$.

Let $\Omega$ be a bounded domain with analytic boundary $\Gamma$. Denote by $\gamma_{c}(z)$ a conformal map from a neighborhood of the unit circle $C \subset \mathbb{C}$ into a neighborhood of $\Gamma$, such that $\gamma_{c}\left(e^{i \theta}\right) \in \Gamma$ for $\theta \in[0,2 \pi]$. We first consider the case of the acoustic single layer potential. For $\phi \in L^{2}(\Gamma)$ we have

$$
[S \phi](x)=\int_{\Gamma} g(x, y) \phi(y) d s(y)
$$

with the Green's function $g(x, y):=\frac{i}{4} H_{0}^{(1)}(k|x-y|)$.

By a change of variables onto the unit circle $C$ we obtain

$$
[S \phi](x)=\int_{C} g\left(\gamma_{c}(w), \gamma_{c}(v)\right) \phi\left(\gamma_{c}(v)\right)\left|\gamma_{c}^{\prime}(v)\right| d s(v),
$$

where $x=\gamma_{c}(w)$ and $y=\gamma_{c}(v)$. Similar to Section 4 we remove the scaling $\left|\gamma_{c}^{\prime}(v)\right|$ by defining the multiplication operator $F$ by $[F \phi](x):=\left|\gamma_{c}^{\prime}(w)\right| \phi(x)$. If we denote the single layer potential operator on the unit circle as $S_{C}$ and let $\phi^{(c)}(v):=\phi\left(\gamma_{c}(v)\right) \in L^{2}(C)$ we obtain

$$
\left[\left(S F^{-1}-S_{C}\right) \phi^{(c)}\right](w)=\int_{C}\left(g\left(\gamma_{c}(w), \gamma_{c}(v)\right)-g(w, v)\right) \phi^{(c)}(v) d s(v) .
$$

Using (NIST Digital Library, eq. 10.4.3,10.8.2) $g(x, y)$ has the representation

$$
\begin{aligned}
g(x, y) & =\frac{i}{4} J_{0}(k|x-y|)-\frac{1}{4} Y_{0}(k|x-y|) \\
& =-\frac{1}{2 \pi} \log (k|x-y|) J_{0}(k|x-y|)+h(x, y),
\end{aligned}
$$

where

$$
\begin{aligned}
h(x, y) & :=\frac{i}{4} J_{0}(k|x-y|)-\frac{1}{2 \pi}(\gamma-\log 2) J_{0}(k|x-y|) \\
& -\frac{1}{2 \pi}\left(\frac{\frac{(k|x-y|)^{2}}{4}}{(1 !)^{2}}-\left(1+\frac{1}{2}\right) \frac{\left(\frac{(k|x-y|)^{2}}{4}\right)^{2}}{(2 !)^{2}}+\left(1+\frac{1}{2}+\frac{1}{3}\right) \frac{\left(\frac{(k|x-y|)^{2}}{4}\right)^{3}}{(3 !)^{2}}-\ldots\right) .
\end{aligned}
$$



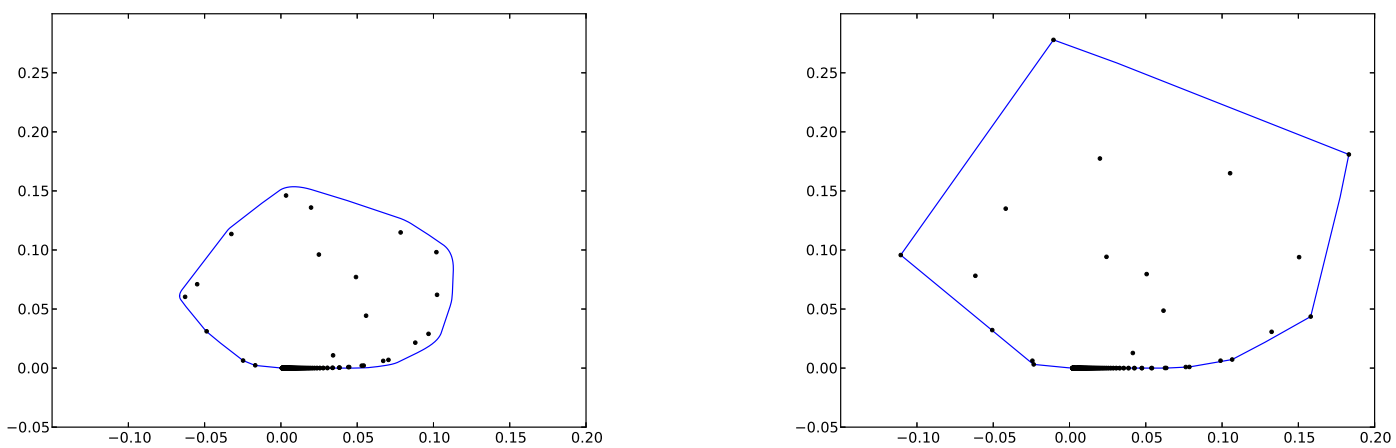

(a) Single Layer Potential
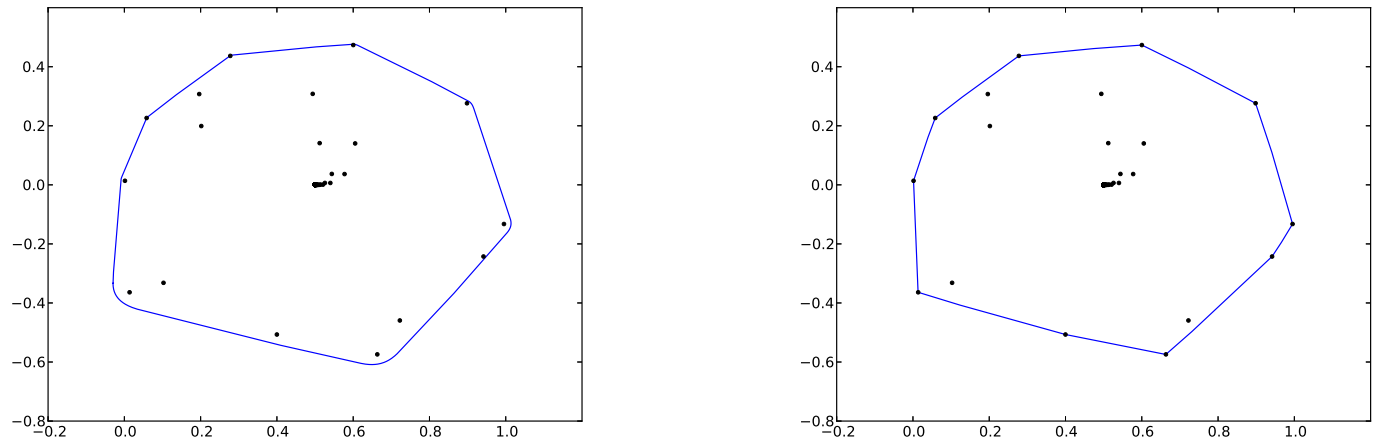

(b) Double Layer Potential
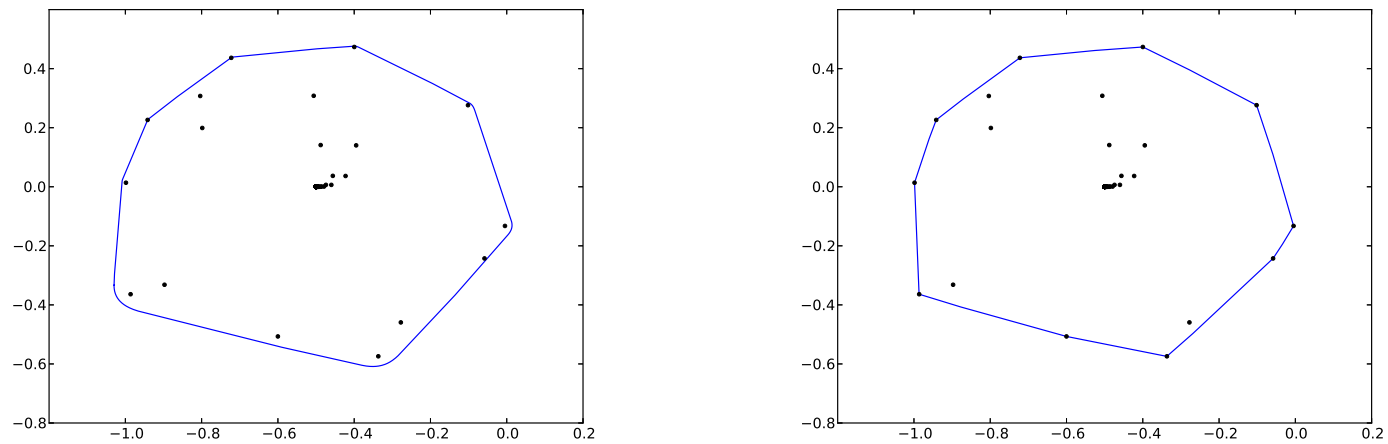

(c) Conjugate Double Layer Potential

FIG. 5: Eigenvalues and numerical ranges of the operators $S, K$ and $T$ in $L^{2}(\Gamma)$ (left plots) and the corresponding eigenvalues and numerical ranges of the operators $S F^{-1}, K$ and $F T F^{-1}$ in $L^{2}\left(F^{-1}, \Gamma\right)$ (right plots). The domain is an ellipse with $a=1$ and $\mu=0.3$. The wavenumber is $k=10$. 
Here, $\gamma$ is Euler's constant (NIST Digital Library, eq. 5.2.3). The function $J_{n}(z)$ has the expansion (NIST Digital Library, eq. 10.2.2)

$$
J_{n}(z)=\left(\frac{z}{2}\right)^{n} \sum_{j=0}^{\infty}(-1)^{j} \frac{\left(\frac{z^{2}}{4}\right)^{j}}{j !(n+j) !} .
$$

It follows that $h(x, y)$ is real analytic in $x$ and $y$. We can now calculate

$$
\begin{aligned}
s(w, v) & :=g\left(\gamma_{c}(w), \gamma_{c}(v)\right)-g(w, v) \\
& =-\frac{1}{2 \pi}\left(\log \left(k\left|\gamma_{c}(w)-\gamma_{c}(v)\right|\right) J_{0}\left(k\left|\gamma_{c}(w)-\gamma_{c}(v)\right|\right)-\log (k|w-v|) J_{0}(k|w-v|)\right) \\
& +h\left(\gamma_{c}(w), \gamma_{c}(v)\right)-h(w, v) .
\end{aligned}
$$

Together with (5.1) we obtain

$$
\begin{aligned}
s(w, v) & =-\frac{1}{2 \pi} \sum_{j=0}^{\infty} \frac{\left(-\frac{\left(k^{2}\right)}{4}\right)^{j}}{(j !)^{2}}\left[\left|\gamma_{c}(w)-\gamma_{c}(v)\right|^{2 j} \log \left(k\left|\gamma_{c}(w)-\gamma_{c}(v)\right|\right)-|w-v|^{2 j} \log (k|w-v|)\right] \\
& +h\left(\gamma_{c}(w), \gamma_{c}(v)\right)-h(w, v) \\
= & :-\frac{1}{2 \pi} \sum_{j=0}^{\infty} \frac{\left(-\frac{\left(k^{2}\right)}{4}\right)^{j}}{(j !)^{2}} \ell_{j}(w, v)+h\left(\gamma_{c}(w), \gamma_{c}(v)\right)-h(w, v)
\end{aligned}
$$

Define the difference quotient $q(w, v):=\frac{\left|\gamma_{c}(w)-\gamma_{c}(v)\right|}{|w-v|}$. Since $\gamma_{c}$ is holomorphic, $q$ is real analytic with respect to $w$ and $v$ with a removable singularity at $w=v$. We can reformulate

$$
\log \left(k\left|\gamma_{c}(w)-\gamma_{c}(v)\right|\right)=\log (k|w-v|)+\log q(w, v),
$$

where $\log q(w, v)$ is real analytic with respect to $w$ and $v$ with limit value $\log \left|\gamma^{\prime}(w)\right|$ for $w=v$ (note that $\gamma^{\prime}(w) \neq 0$ for $\left.w \in C\right)$. We obtain

$$
\begin{aligned}
\ell_{j}(w, v) & =|w-v|^{2 j} \log (|w-v|)\left[q(w, v)^{2 j}-1\right]+\left|\gamma_{c}(w)-\gamma_{c}(v)\right|^{2 j} \log q(w, v) \\
& +|w-v|^{2 j}\left[q(w, v)^{2 j}-1\right] \log k
\end{aligned}
$$

For $j=0$ the singular term in $\ell_{0}$ disappears. For $j>0$ the $\ell_{j}$ are continuous in $w$ and $v$. We summarize the results in the following lemma.

Lemma 5.1 Similar to Section 4 define the multiplication operator $F$ by $[F \phi](x)=\left|\gamma_{c}^{\prime}(w)\right| \phi(x)$, where $x=\gamma_{c}(w)$. Let $S$ be the single layer potential operator defined in Section 1 on the boundary $\Gamma$, and let $S_{C}$ be the corresponding operator defined on the unit circle $\Gamma_{C}$. Define $\phi^{(c)}(x)=\phi\left(\gamma_{c}(x)\right)$ for $\phi \in L^{2}(\Gamma)$. Then it holds that

$$
\left[S F^{-1} \phi\right](x)=\left[S_{C} \phi^{(c)}\right](w)+\left[\hat{S} \phi^{(c)}\right](w),
$$

where

$$
\left[\hat{S} \phi^{(c)}\right](w)=\int_{\Gamma_{C}} s(w, v) \phi^{(c)}(v) d s(v)
$$

$\hat{S}$ is a compact operator on $L^{2}(\Gamma)$ with continuous kernel $s(w, v)$.

For $x=\gamma_{c}(w)$ with $w=e^{i \theta}$ for some $\theta \in[0,2 \pi)$ consider the function $u_{n}(x):=u_{n}^{(c)}(w) \in L^{2}(\Gamma)$ with $u_{n}^{(c)}(w)=w^{n}$, which is the $n$th Fourier mode transplanted to $\Gamma$ and let $F$ be the multiplication operator defined in Lemma 5.1. Then together with Theorem 1.1 we have

$$
\left[S F^{-1} u_{n}\right](x)=\left[S_{C} u_{n}^{(c)}\right](w)+\left[\hat{S} u_{n}^{(c)}\right](w)=\lambda_{n}^{(S)} u_{n}(x)+\left[\hat{S} u_{n}^{(c)}\right](w) .
$$


We now estimate the remainder term $\hat{S} u_{n}^{(c)}$. It has the form

$$
\left[\hat{S} u_{n}^{(c)}\right](w)=\int_{0}^{2 \pi} s(w, v(\theta)) e^{i n \theta} d \theta
$$

where $v(\theta)=[\cos \theta, \sin \theta]^{T}$ is a parameterisation of the unit circle. Hence, $\hat{S} u_{n}^{(c)}$ is the $-n$th Fourier mode of $s(w, v(\theta))$ with respect to $\theta$. The decay of the Fourier coefficents is determined by the smoothness of $s$.

LEMma 5.2 The Fourier coefficients $\hat{s}_{n}(w)$ of $s(w, v(\theta))$ with respect to $\theta$ satisfy $\hat{s}_{n}(w)=\mathscr{O}\left(|n|^{-3}\right)$ where the omitted constant is independent of $w \in C$, where $C$ is the unit circle.

Proof. From (5.3) it follows that

$$
s(w, v(\theta))=\frac{1}{2 \pi}\left(\frac{k^{2}}{4}\right)|w-v(\theta)|^{2} \log (|w-v(\theta)|)\left[q(w, v(\theta))^{2}-1\right]+\cdots,
$$

where we have written down only the most singular term. Since $w$ and $v(\theta)$ both are variables on the unit circle we have that

$$
|w-v(\theta)|^{2}=2-2 \cos \left(\theta-\theta_{w}\right)
$$

where $w=e^{i \theta_{w}}$ in complex coordinates. Thus

$$
s(w, v(\theta))=\frac{1}{2 \pi}\left(\frac{k^{2}}{4}\right)\left(1-\cos \left(\theta-\theta_{w}\right)\right) \log \left(2-2 \cos \left(\theta-\theta_{w}\right)\right)\left[q(w, v(\theta))^{2}-1\right]+\ldots .
$$

Differentiating with respect to $\theta$ gives

$$
\begin{aligned}
\frac{\partial s(w, v(\theta))}{\partial \theta} & =\frac{1}{2 \pi}\left(\frac{k^{2}}{4}\right)\left[\sin \left(\theta-\theta_{w}\right) \log \left(2-2 \cos \left(\theta-\theta_{w}\right)\right)+\sin \left(\theta-\theta_{w}\right)\right]\left[q(w, v(\theta))^{2}-1\right] \\
& +\ldots \\
\frac{\partial^{2} s(w, v(\theta))}{\partial \theta^{2}} & =\frac{1}{2 \pi}\left(\frac{k^{2}}{4}\right)\left[\cos \left(\theta-\theta_{w}\right) \log \left(2-2 \cos \left(\theta-\theta_{w}\right)\right)+1+2 \cos \left(\theta-\theta_{w}\right)\right]\left[q(w, v(\theta))^{2}-1\right] \\
& +\ldots
\end{aligned}
$$

Using the Taylor expansion of $\gamma_{c}$ we have

$$
\left|\gamma_{c}(w)-\gamma_{c}(v)\right|^{2}=\left|\gamma_{c}(w)(v-w)+\mathscr{O}\left(|v-w|^{2}\right)\right|^{2}=\left|\gamma^{\prime}(w)\right|^{2}|v-w|^{2}+\mathscr{O}\left(|v-w|^{3}\right)
$$

and therefore $q^{2}(w, v)=\left|\gamma_{c}^{\prime}(w)\right|^{2}+\mathscr{O}(|v-w|)$ as $v \rightarrow w$. Using this and (5.5) it follows that

$$
\frac{\partial^{2} s(w, v(\theta))}{\partial \theta^{2}}=\frac{1}{\pi}\left(\frac{k^{2}}{4}\right) \log |w-v(\theta)|\left[\left|\gamma_{c}^{\prime}(w)\right|^{2}-1\right]+\ldots
$$

where we have omitted terms of order $\mathscr{O}\left(|w-v(\theta)|^{j} \log |w-v(\theta)|\right)$ for $j>0$ and analytic terms. For the Fourier-coefficients $\hat{s}_{n}$ we now have, using integration by parts,

$$
\hat{s}_{-n}(w)=\left(\frac{i}{n}\right)^{2} \int_{0}^{2 \pi} \frac{\partial^{2} s(w, v(\theta))}{\partial \theta^{2}} e^{i n \theta} d \theta .
$$

By (5.7) it follows that up to higher order terms with faster decaying Fourier coefficients the integrand is a multiple of the $-n$th Fourier coefficient of the kernel of the Laplace single layer potential on the unit circle, which decays like $\mathscr{O}\left(|n|^{-1}\right)$ independently of $w \in C$ (Betcke et al., 2012, Appendix A). It follows that $\hat{s}_{n}(w)=\mathscr{O}\left(|n|^{-3}\right)$.

Combining Lemma 5.1 and Lemma 5.2 it follows from

$$
\left[\hat{S} u_{n}^{(c)}\right](w)=\hat{s}_{-n}(w)
$$

that

$$
S F^{-1} u_{n}=\lambda_{n}^{(S)} u_{n}+\mathscr{O}\left(|n|^{-3}\right),
$$


where the eigenvalues $\lambda_{n}^{(S)}$ are the circle eigenvalues defined in Theorem 1.1.

We note that this result does not necessarily imply that the eigenvalues on general domains converge to the eigenvalues on the unit circle for $n \rightarrow \infty$. The reason is that for nonnormal operators a residual of size $\varepsilon$ for an approximate eigenpair only implies that the corresponding approximate eigenvalue is in the $\varepsilon$-pseudospectrum (see the third characterisation of pseudospectra in Definition 2.1). But this can be a large set depending on the nonnormality of the operator. It is nevertheless useful to compare the result in Theorem 5.8 to the decay of the eigenvalues $\lambda_{n}^{(S)}$ on the unit circle. In (Betcke et al., 2012, Appendix A) it is shown that $\lambda_{n} \sim \frac{1}{2|n|}$. Hence, the cubic decay of the residual in (5.8) is nontrivial.

For the operators $K$ and $T$ similar results with the same cubic decay of the residual can be derived. The details are given Betcke et al. (2012). We summarize the main result of this section in the following Theorem.

TheOREM 5.1 Using the notation of Lemma 5.1, the scaled Single Layer Potential $S F^{-1}$ approximately diagonalizes in a scaled Fourier basis $u_{n}(x)=w^{n}$, where $x=\gamma_{c}(w)$, such that

$$
\begin{aligned}
S F^{-1} u_{n} & =\lambda_{n}^{(S)} u_{n}+\mathscr{O}\left(|n|^{-3}\right), \\
K u_{n} & =\lambda_{n}^{(K)} u_{n}+\mathscr{O}\left(|n|^{-3}\right), \\
F T F^{-1} u_{n} & =\lambda_{n}^{(T)} u_{n}+\mathscr{O}\left(|n|^{-3}\right),
\end{aligned}
$$

where the eigenvalues $\lambda_{n}^{(S)}, \lambda_{n}^{(K)}$, and $\lambda_{n}^{(T)}$ are the circle eigenvalues defined in Theorem 1.1.

For the operators $K$ and $T$ it is shown in (Betcke et al., 2012, Appendix A) that the eigenvalues decay like

$$
\begin{aligned}
& \lambda_{n}^{(K)} \sim \frac{1}{2}+\left(\frac{k^{2}}{4}\right)|n|^{-3}, \\
& \lambda_{n}^{(T)} \sim-\frac{1}{2}+\left(\frac{k^{2}}{4}\right)|n|^{-3} .
\end{aligned}
$$

Hence, the order of the decay of the eigenvalues is the same as the decay of the residual in Theorem 5.1. The results for the $K$ and $T$ therefore appear to be weaker than the results for $S$.

We conclude this section with a simple numerical example. The domain is an ellipse defined by $\mu=0.3$ and $a=1$. We discretise the operators $S F^{-1}, K$, and $F T F^{-1}$ using a Galerkin discretisation in $L^{2}\left(F^{-1}, \Gamma\right)$; this is the appropriate space for the scaled Fourier modes to be orthogonal. After discretisation we obtain the finite dimensional eigenvalue problem $\mathbf{B} x=\lambda \mathbf{M}^{\left(F^{-1}\right)} x$, where $\mathbf{M}^{\left(F^{-1}\right)}$ is the mass matrix in the $L^{2}\left(F^{-1}, \Gamma\right)$ inner product and $\mathbf{B}$ is the discretisation of either of $S F^{-1}, K$, or $F T F^{-1}$ in this inner product. Let $\mathbf{U}$ be a matrix of discretised Fourier modes in the given finite dimensional basis. Here, we choose Fourier modes from $N=-200$ to $N=200$. According to the results of this section $\mathbf{U}$ approximately diagonalises the pencil $\left(\mathbf{B}, \mathbf{M}^{\left(F^{-1}\right)}\right)$, in the sense that, ignoring discretisation errors, for the columns $\mathbf{R}_{j_{n}}$ of

$$
\mathbf{R}:=\mathbf{U}^{H} \mathbf{B} \mathbf{U}-\mathbf{U}^{H} \mathbf{M}^{\left(F^{-1}\right)} \mathbf{U} \Lambda,
$$

where $j_{n}$ is the column index of the $n$th discretised Fourier mode in $\mathbf{U}$, we have

$$
\mathbf{R}_{j_{n}}=\mathscr{O}\left(|n|^{-3}\right),
$$

Here, $\Lambda$ is the diagonal matrix of circle eigenvalues of either the single, double, or conjugate double layer potential associated with the Fourier modes in $\mathbf{U}$. The decay of the columns $\mathbf{R}_{j_{n}}$ for the wavenumber $k=10$ is shown in Figure 6. It is interesting to note that the convergence initially stagnates for $|n|<10$. It seems that the approximation results of this section only become sharp for the asymptotic regime as $|n|>k$.

Finally we remark that repeating the above analysis for the Laplace single, double, and conjugate double layer potentials shows exponential decay of the residual when approximating eigenpairs on general domains with eigenpairs on the circle (as opposed to the algebraic decay above for the Helmholtz case). 


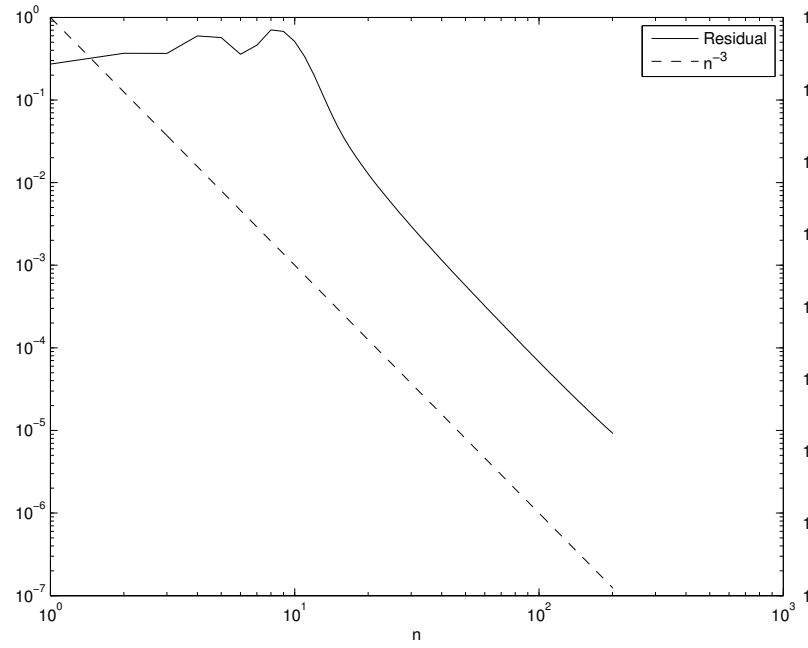

(a) Single Layer

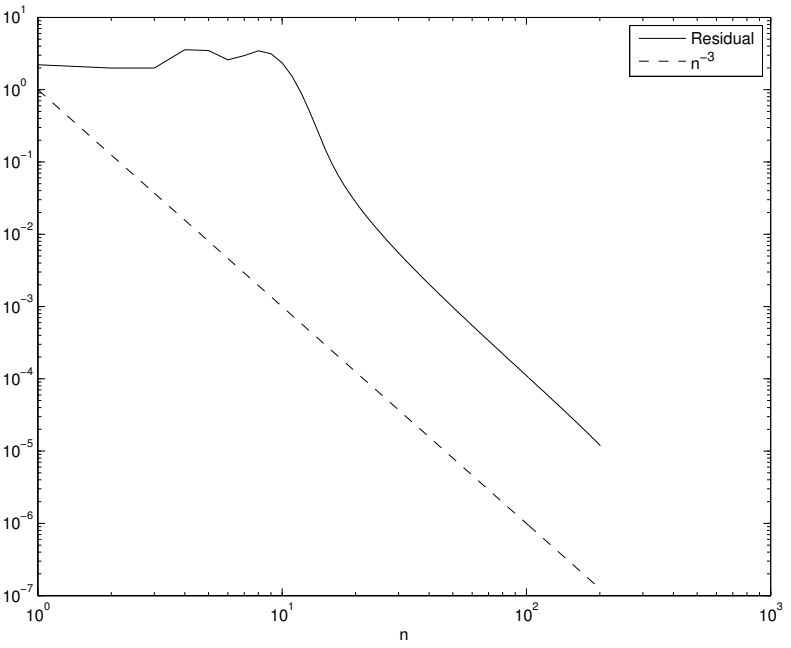

(b) Double Layer

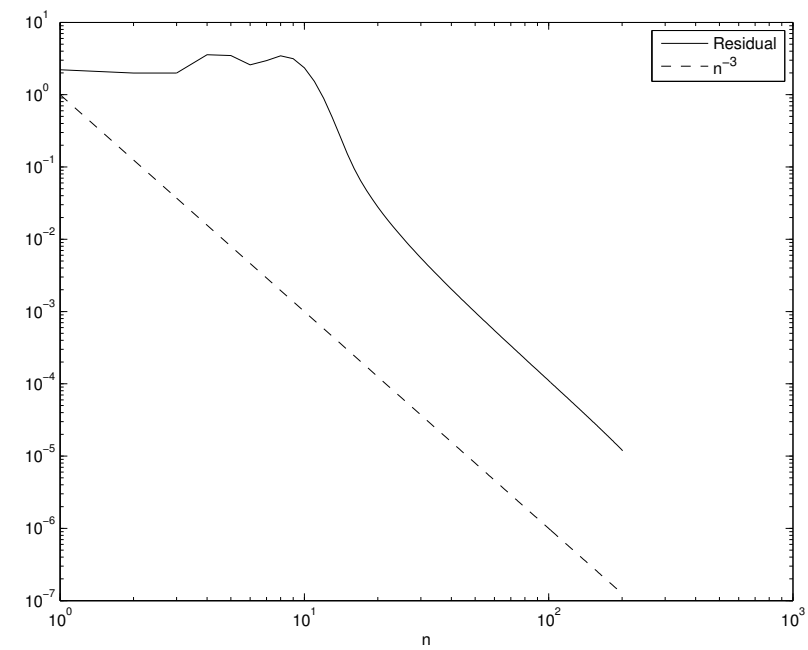

(c) Conjugate double layer

FIG. 6: Cubic decay of the residual for the single layer, double layer, and conjugate double layer potential. 
The reason for this is that the difference between the Laplace Green's functions

$$
\frac{-1}{2 \pi} \log \left|\gamma_{c}(x)-\gamma_{c}(y)\right|-\left(-\frac{1}{2 \pi}\right) \log |x-y|=-\frac{1}{2 \pi} \log \frac{\left|\gamma_{c}(x)-\gamma_{c}(y)\right|}{|x-y|}
$$

has a removable singularity for $x=y$ and is therefore analytic in $x$ and $y$, giving exponential decay of the corresponding Fourier coefficients. (Recall that in the Helmholtz case the difference $s(w, v)$ is not analytic - see (5.4) - hence the algebraic decay.)

\section{Spectral decompositions and nonnormality of the combined potential operator}

In Section 1 we introduced the combined potential operator $A_{\eta}: L^{2}(\Gamma) \rightarrow L^{2}(\Gamma)$. In this section we will demonstrate how this operator can be modified to also fit into the framework of the decompositions derived in Section 4 and 5 and present numerical examples that demonstrate the effect of these modifications on the normality of the operator. In order to derive the modified combined potential operator we briefly recap the derivation of the combined potential operator. Consider the problem of time-harmonic acoustic scattering from a sound-soft bounded obstacle $\Omega \subset \mathbb{R}^{d},(d=2,3)$ with Lipschitz boundary $\Gamma:=\partial \Omega$. That is, we are looking for the solution $u$ of the problem

$$
\begin{array}{rlll}
\Delta u+k^{2} u & = & 0 & \text { in } \mathbb{R}^{d} \backslash \bar{\Omega}, \\
u & = & 0 & \text { on } \Gamma, \\
\frac{\partial u_{s}}{\partial r}-i k u_{s} & = & o\left(r^{-(d-1) / 2}\right),
\end{array}
$$

where $u=u_{i n c}+u_{s}$ is the total field, $u_{\text {inc }}$ is an entire solution of (6.1), such as an incident plane wave, $u_{s}$ is the scattered field, $r$ is the radial coordinate, and $k>0$ is the wavenumber. With the standard free-space Green's function

$$
g(x, y)=\frac{i}{4} H_{0}^{(1)}(k|x-y|)
$$

for $x, y \in \mathbb{R}^{2}, x \neq y$, the solution $u$ is given by

$$
u(x)=u_{\text {inc }}(x)-\int_{\Gamma} g(x, y) \partial_{n} u(y) d s(y), \quad x \in \mathbb{R}^{d} \backslash \bar{\Omega},
$$

where $\partial_{n} u$ is the outward pointing normal derivative of $u$. Taking the normal trace $\gamma_{1}^{+}$of (6.4) and recalling the definition of the boundary integral operator $T$ from Section 1 gives

$$
(I+T) \partial_{n} u=\frac{\partial}{\partial n} u_{i n c} .
$$

Furthermore, taking the trace $\gamma_{0}^{+}$of (6.4) and using the boundary condition (6.2) it follows that

$$
S \partial_{n} u=u_{\text {inc }} .
$$

Multiplying (6.6) with $i \eta$ for $\eta \neq 0$ and subtracting from (6.5) gives the operator equation

$$
(I+T-i \eta S) \partial_{n} u=\frac{\partial}{\partial n} u_{i n c}-i \eta u_{i n c} .
$$

The standard combined operator is defined as $A_{\eta}:=2(I+T-i \eta S)$, where the factor 2 is frequently included so that $A_{\eta}$ is a perturbation of the identity (as opposed to $\frac{1}{2} I$ ). To apply the results from Section 4 and 5 we use the multiplication operator $F$ defined in Lemma 5.1. Multiplying (6.6) by $i \eta F^{-1}$ and subtracting from (6.5) gives

$$
\frac{1}{2} A_{\eta}^{(F)} u_{n}:=\left(I+T-i \eta F^{-1} S\right) u_{n}=\frac{\partial}{\partial n} u_{i n c}-i \eta F^{-1} u_{i n c} .
$$

In Chandler-Wilde \& Langdon (2007) it was shown that, for general Lipschitz $\Gamma, A_{\eta}$ is a bounded operator with bounded inverse on $L^{2}(\Gamma)$ for every $\eta \neq 0$ and $k>0$. Since $F$ is bounded with bounded inverse the same arguments show that $A_{\eta}^{(F)}$ is bounded with a bounded inverse on $L^{2}(\Gamma)$. However, by rescaling $S$ with $F^{-1}$ we can now give a simple approximate eigenvalue decomposition for a bounded domain $\Omega$ with analytic boundary $\Gamma$. 
Theorem 6.1 Define $F$ as in Lemma 5.1 and let the boundary $\Gamma$ be analytic. Denote by $u_{n}$ the $n$th scaled Fourier mode as defined in Theorem 5.1. The operator $F A_{\eta}^{(F)} F^{-1}$ admits an approximate eigendecomposition of the form

$$
F A_{\eta}^{(F)} F^{-1} u_{n}=\lambda_{n}^{(A)} u_{n}+\mathscr{O}\left(|n|^{-3}\right),
$$

for any $n \neq 0$, where $\lambda_{n}^{(A)}$ is defined in Theorem 1.1.

Proof. We have

$$
F A_{\eta}^{(F)} F^{-1}=2\left(I+F T F^{-1}+S F^{-1}\right) .
$$

Applying Theorem 5.1 now gives the desired result.

The asymptotics in (Betcke et al., 2012, Appendix A) show that the eigenvalues $\lambda_{n}^{(A)}$ behave asymptotically like $1+\mathscr{O}\left(|n|^{-1}\right)$. Thus, the statement of (6.1) is nontrivial as it states that the eigenpairs on the circle approximate the eigenpairs of the combined potential operator on general smooth domains up to a cubically small error.

Instead of the general approximate eigenvalue decomposition given in Theorem 6.1 we can also use the exact eigenvalue decompositions of the operators $S F^{-1}$ and $F T F^{-1}$ on the ellipse given in Theorem 4.2 to obtain an exact eigenvalue decomposition of the combined potential operator on ellipses; we omit the details.

We now visualise the approximate decomposition of the combined potential using two examples, an ellipse and the crescent domain from Section 2. We compare the exact and approximate eigenvalue decomposition of the combined potential operator $A_{\eta}^{(F)}$ for growing $k$. We always choose $\eta=k$, which is a standard choice for sufficiently large wavenumbers (Betcke et al., 2011).

After a change of variables the eigenvalue problem considered in Theorem 6.1 becomes

$$
A_{\eta}^{(F)} \tilde{u}=\lambda \tilde{u},
$$

where $\tilde{u}=F^{-1} u$. Theorem 5.1 showed that we have an approximate eigendecomposition if the $u_{n}$ are scaled Fourier modes. These functions are orthogonal in $L^{2}\left(F^{-1}, \Gamma\right)$. After the change of variables $\tilde{u}_{n}=F^{-1} u_{n}$ the functions $\tilde{u}$ are orthogonal in $L^{2}(F, \Gamma)$. The variational formulation of (6.7) associated with this inner product is to find nontrivial pairs $(\lambda, u) \in \mathbb{C} \times L^{2}(F, \Gamma)$, such that

$$
\left(F A_{\eta}^{(F)} u, v\right)_{L^{2}(\Gamma)}=\lambda(F u, v)_{L^{2}(\Gamma)} \forall v \in L^{2}(F, \Gamma) .
$$

Let $\mathbf{A}_{\eta}^{(F)} x=\lambda \mathbf{M}^{(F)} x$ be the finite dimensional Galerkin discretisation in $L^{2}(F, \Gamma)$ associated with this problem. Let $\mathbf{U}$ be the matrix whose columns are the coefficients of the Galerkin projections of the functions $\tilde{u}_{n}$ in $L^{2}(F, \Gamma)$. Then from Theorem 6.1 we expect that $\mathbf{U}^{H} \mathbf{A}_{\eta}^{(F)} \mathbf{U}$ is approximately diagonalised with an error of order $O\left(|n|^{-3}\right)$ if we ignore discretization effects. Also, by the $L^{2}(F, \Gamma)$ orthogonality of the basis functions, the matrix $\mathbf{U}^{H} \mathbf{M}^{(F)} \mathbf{U}$ is diagonal up to discretisation errors.

Figure 7 shows logarithmic plots of $\left|\mathbf{U}^{H} \mathbf{A}_{\eta}^{(F)} \mathbf{U}\right|$ for an ellipse with $a=1$ and $\mu=.3$ (left plots) and the crescent domain from Section 2 (right plots). The top plots show the case $k=10$ and the bottom plots the case $k=50$. The color scale uses white for large values. For the ellipse the operator is almost perfectly diagonalised in a Fourier basis with a small error concentrated at the low-frequency Fourier modes. In the crescent case we still have a strong diagonal, but now the off-diagonal errors are larger than in the ellipse case. This is expected since the conformal map from the unit circle to the crescent has singularities close to the boundary of the domain.

In Figure 8 we plot the relative distance between the diagonal values $\left|\operatorname{diag}\left(\mathbf{A}_{\eta}^{(F)}\right)\right| /\left|\operatorname{diag}\left(\mathbf{M}^{(F)}\right)\right|$ and the associated circle eigenvalues $\lambda_{n}^{(A)}$ for $k=10$ on the crescent domain. After an initial stagnation phase up to approximately $|n|=10$ the diagonal values $\left|\operatorname{diag}\left(\mathbf{A}_{\eta}^{(F)}\right)\right| /\left|\operatorname{diag}\left(\mathbf{M}^{(F)}\right)\right|$ are well approximated by circle eigenvalues. Figure 9 shows the diagonal values of $\left|\operatorname{diag}\left(\mathbf{A}_{\eta}^{(F)}\right)\right| /\left|\operatorname{diag}\left(\mathbf{M}^{(F)}\right)\right|$ for the crescent and the absolute values of the circle eigenvalues $\lambda_{n}^{(A)}$ in the case $k=10$. The eigenvalues are oscillatory for $|n|<10$. After a short transition zone they enter the asymptotic regime for $|n|>10$ in which the circle 

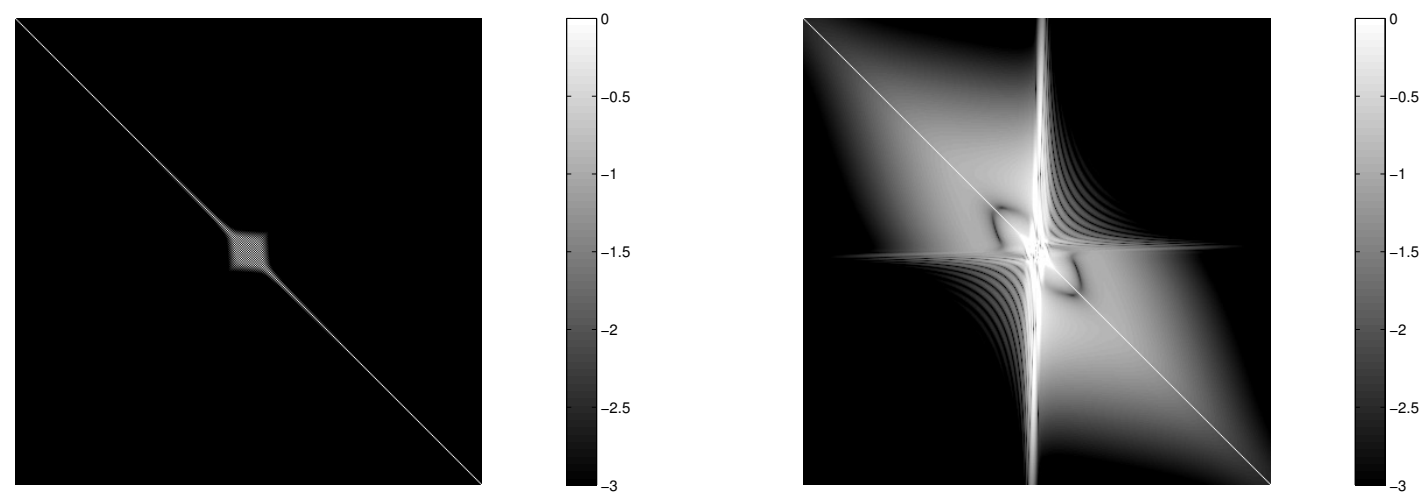

(a) $\mathrm{k}=10$
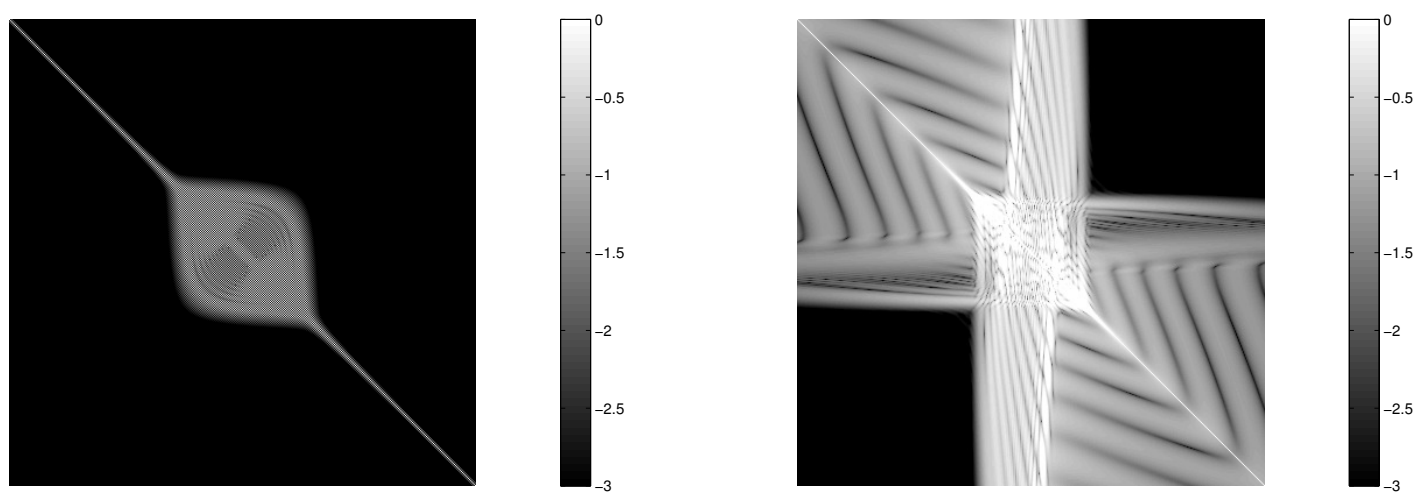

(b) $\mathrm{k}=50$

FIG. 7: Logarithmic plots of $\left|\mathbf{U}^{H} \mathbf{A}_{\eta}^{(F)} \mathbf{U}\right|$ for an ellipse with parameters $a=1, \mu=3$ (left) and the crescent domain from Section 2 (right). The upper plots are for $k=10$ and the bottom plots for $k=50$. In both cases we display results for scaled Fourier modes from $n=-200$ to $n=200$. 


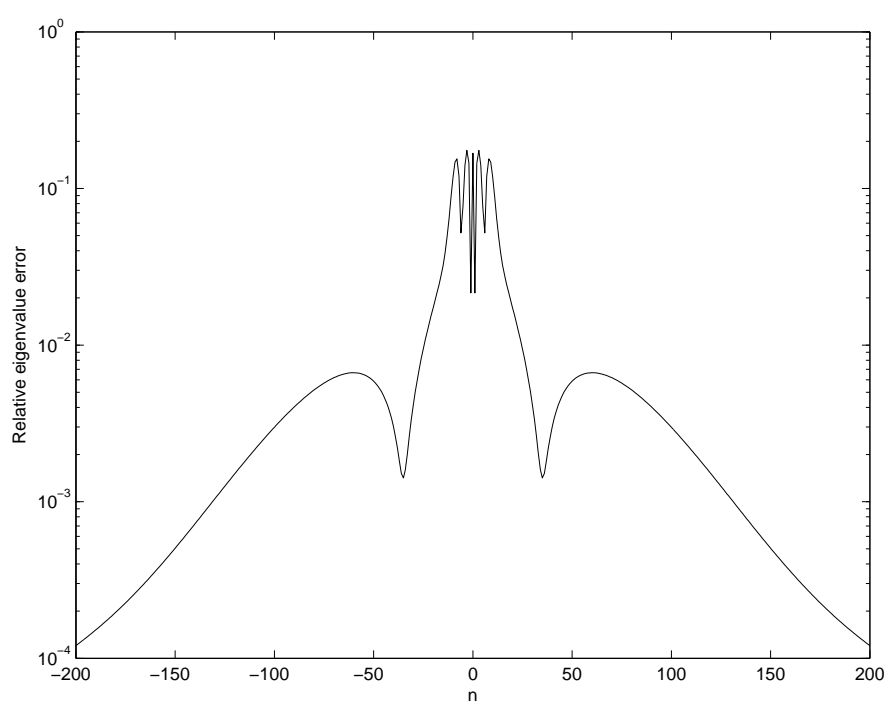

FIG. 8: Relative distance of the diagonal values $\left|\operatorname{diag}\left(\mathbf{A}_{\eta}^{(F)}\right)\right| /\left|\operatorname{diag}\left(\mathbf{M}^{(F)}\right)\right|$ to the associated circle eigenvalues $\lambda_{n}^{(A)}$ on the crescent domain with wavenumber $k=10$.

eigenvalues behave like $1+\mathscr{O}\left(|n|^{-1}\right)$. The numerical results indicate that the approximations via circle eigenvalues are valid in this asymptotic regime.

We already showed the pseudospectrum for the combined potential operator $A_{\eta}$ in the standard $L^{2}(\Gamma)$ inner product for the crescent domain and wavenumber $k=10$ in Figure 2. In Figure 10 we compare the pseudospectrum and numerical range of $A_{\eta}$ in $L^{2}(\Gamma)$ (top figure) with that of $A_{\eta}^{(F)}$ in $L^{2}(F, \Gamma$ ) (bottom figure) for the crescent domain and wavenumber $k=50$. In both cases the dashed line denotes the boundary of the numerical range. The pseudospectra and numerical range of the operator $A_{\eta}^{(F)}$ in $L^{2}(F, \Gamma)$ are approximated by those of the matrix $\mathbf{C}^{-H} \mathbf{A}_{\eta}^{(F)} \mathbf{C}^{-1}$, where $\mathbf{C}^{H} \mathbf{C}=\mathbf{M}^{(F)}$ is the Cholesky decomposition of $\mathbf{M}^{(F)}$. This corresponds to changing to a unitary basis of the finite dimensional Galerkin subspace of $L^{2}(F, \Gamma) .^{2}$

Most of the eigenvalues in the pre-asymptotic regime cluster around the value 2 in the complex plane. For the scaled operator $A_{\eta}^{(F)}$ nonnormality seems to manifest itself mostly around these eigenvalues; this can be seen, for example, in the large boundary of the $10^{-1}$-pseudospectrum, and also in the fact that the $10^{-2}$-pseudospectrum is visible close to 2 on this plotting scale. The large eigenvalues in the transitional regime have only small circular $10^{-1}$-pseudospectra. They behave almost like eigenvalues of a normal operator. The picture looks different for the unscaled operator $A_{\eta}$, where the pseudospectral sets are much larger in the pre-asymptotic and transitional regime. However, in both cases the overall nonnormality seems still quite mild. Even for the pre-asymptotic eigenvalues in the cluster around 2 the eigenvalue condition number computed by EigTool is only of the order $10 .^{3}$

Interestingly, the numerical range includes the origin for the case of $A_{\eta}^{(F)}$, but not for $A_{\eta}$. Thus, although rescaling $A_{\eta}$ has meant that its spectrum has become closer to that of the circle (in the sense of Theorem

\footnotetext{
${ }^{2}$ In the same way, to approximate the pseudospectrum and numerical range of $A_{\eta}$ in $L^{2}(\Gamma)$ we use the matrix $\mathbf{C}^{-H} \mathbf{A}_{\eta} \mathbf{C}^{-1}$, where $\mathbf{A}_{\eta}$ is the discretisation of $A_{\eta}$ in $L^{2}(\Gamma)$ and $\mathbf{C}$ is the Cholesky factor of $\mathbf{M}$, the mass matrix in the standard $L^{2}(\Gamma)$ inner product.

${ }^{3}$ Eigenvalue condition numbers measure the sensitivy of an eigenvalue to perturbations in a matrix $A$. A condition number of 10 means that a perturbation in $A$ of size $\varepsilon$ maximally perturbs the eigenvalue by around $10 \varepsilon$ as $\varepsilon \rightarrow 0$. A precise definition is for example given in (Demmel, 1997).
} 


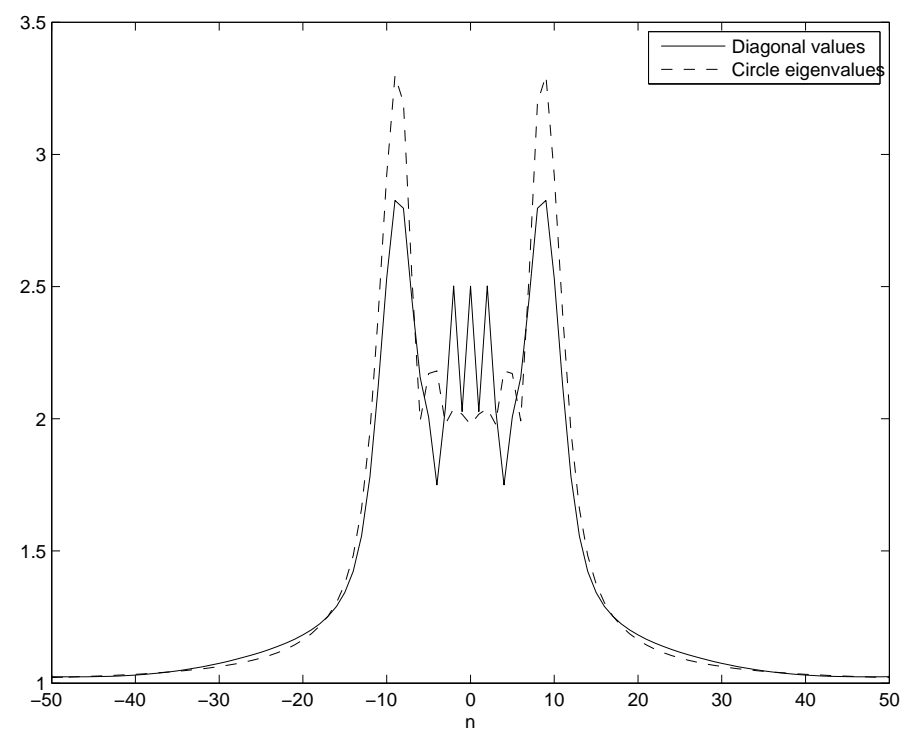

FIG. 9: Plot of $\left|\operatorname{diag}\left(\mathbf{A}_{\eta}^{(F)}\right)\right| /\left|\operatorname{diag}\left(\mathbf{M}^{(F)}\right)\right|$ and the absolute values of the circle eigenvalues $\lambda_{n}^{(A)}$ for $k=10$.

6.1), coercivity has been lost.

Finally we note that changing the coupling parameter $\eta$ in the combined potential operator $A_{\eta}$ from a constant to an operator has been proposed in several other contexts (and goes back at least to Panich (1965)). For example, Colton \& Kress (1998) considered $\eta$ as an operator in order to obtain an invertible combined potential operator for the Neumann problem on smooth domains, and Mitrea (1996) for the Dirichlet problem on Lipschitz domains. (Since both these authors considered indirect boundary integral equations, i.e. those not arising from Green's integral representation (6.4), their operator $\eta$ appears on the right of $S$, whereas for direct boundary integral equations, if $\eta$ is an operator it appears on the left, as above.) More recently Buffa \& Hiptmair (2005), Engleder \& Steinbach (2007), and Engleder \& Steinbach (2008) considered $\eta$ as various operators in order to prove quasi-optimality on Lipschitz domains of Galerkin discretisations of the resulting combined potential operator (with the operator acting in the trace spaces). Operator-valued $\eta$ s have also been considered from a more practical point of view, with Anand et al. (2011) and Antoine \& Darbas (2012) interested in obtaining combined potential operators whose discretisations require a small number of iterations when solved with Krylov subspace methods.

\section{Conclusions}

The main results of this paper can be summarized as follows. In the first part of this paper we proved that among all sufficiently smooth domains (at least $C^{2, \alpha}$ ) the operator $S$ is normal if and only if the domain is a ball. This shows that at least for the single layer potential the operator is in general nonnormal. We believe that similar results will hold for $K, T$ and the combined operator $A_{\eta}$, but proofs for these cases are not yet available.

The next result was that on an ellipse an exact eigendecomposition can be derived for the operators $S, K$ and $T$, which also implies that these operators are normal on the ellipse under a suitable scaling in a modified $L^{2}$ inner product. The main tool for this result was the decomposition of the Green's function using separation of variables in elliptical coordinates. There are eleven orthogonal coordinate systems in 3-d in which the Helmholtz equation is separable (Moon \& Spencer, 1971, §1), (Morse \& Feshbach, 1953b, Chapter 5), and analogous decompositions of the Green's function in each of these can be obtained using the general procedure in (Morse \& Feshbach, 1953b, §7.2) (or following the essentially equivalent method of (Betcke et al., 2012, Appendix B)). Thus, for domains that are defined by one coordinate being constant in one of these eleven coordinate systems (such as the circle/sphere in polar coordinates and the ellipse 

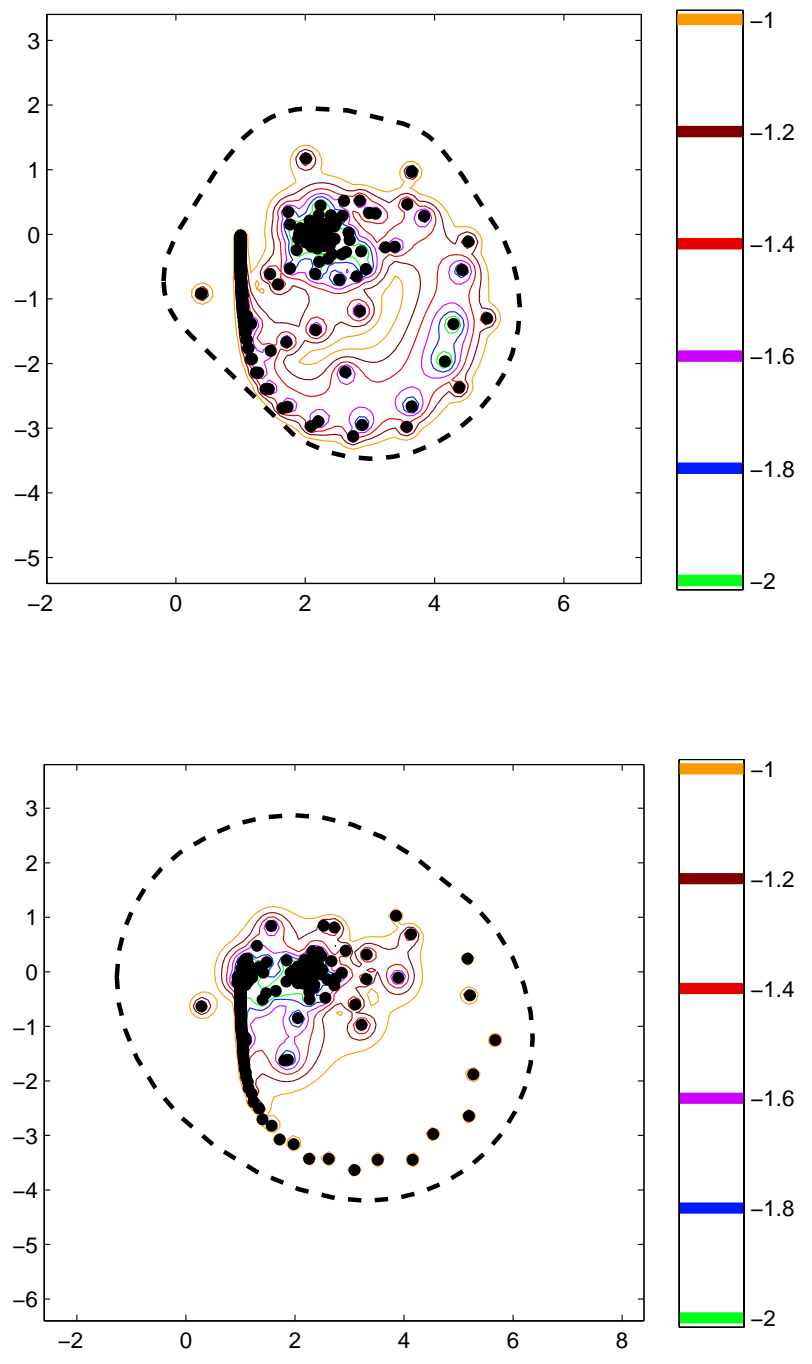

FIG. 10: Pseudospectral plots of $A_{\eta}$ in $L^{2}(\Gamma)$ (top figure) and $A_{\eta}^{(F)}$ in $L^{2}(F, \Gamma)$ (bottom figure) 
in elliptical coordinates) it should be possible to obtain normality of $S, K$, and $T$ under modified inner products.

The final result was an approximate decomposition using circle eigenvalues and scaled Fourier modes on general analytic domains. The numerical experiments in Section 6 indicate that this approximation becomes valid for scaled Fourier modes with number $n$, such that $|n|>k$. Furthermore, the bottom plot of Figure 10 indicates that under the correct scaling the nonnormality is mostly concentrated in the part of the spectrum associated with the first $\mathscr{O}(k)$ eigenvalues in the pre-asymptotic regime.

An interesting byproduct of the approximate decompositions in Section 6 concerns the order of the double layer potential considered as a pseudo-differential operator. It is well-known that the operator $K-\frac{1}{2} I$ in 3-d is order -1 (Hsiao \& Wendland, 2008, p. 518). However, the eigenvalue asymptotics derived in (Betcke et al., 2012, Appendix A) on the circle seem to imply that the order is -3 for the 2-d circle case. Equation (5.9) indicates that the order may even be -3 for general smooth domains in $2-d{ }^{4}$

The next step will be to investigate the behavior of these operators for iterative solvers such as GMRES (see Antoine \& Darbas (2012) for a recent survey). For normal operators GMRES convergence is purely determined by the distribution of the eigenvalues. For nonnormal operators this is not sufficient and techniques such as the numerical range or pseudospectra become important for the analysis. Research into this question is currently ongoing.

Another open question is the high-frequency behavior. The approximate decompositions given in this paper for general domains are valid for sufficiently high Fourier-modes with $|n|>k$. However, for highfrequency scattering problems it is important to know what happens for $k$ very large. The analysis presented in this paper does not provide much insight into this case and one would need to develop high-frequency approximations. Some previous work in this direction is that of Warnick \& Chew (2001) who considered solving the boundary value problem of scattering by a crack using the single layer potential $S$. They decomposed $S$ into a normal part and a non-normal part. The normal part is related to the geometric optics approximation (valid for large $k$ ), which can be understood as replacing the crack with an infinite line. This decomposition was obtained using the exact spectral decomposition of $S$ for this particular domain, which in turn relied on the analogue of (1.2) in cartesian coordinates.

\section{Acknowledgements}

We would like to thank the anonymous referees for their very helpful comments that improved the manuscript. In particular, we would like to thank one anonymous referee for pointing out the result of Corollary 4.2 to us.

\section{References}

ANAND, A., Ovall, J. \& TurC, C. (2011) Well conditioned boundary integral equations for twodimensional sound-hard scattering problems in domains with corners. Journal Integral Equations and Applications.

Antoine, X. \& DARbAS, M. (2012) Integral equations and iterative schemes for acoustic scattering problems. Numerical Methods for Acoustics Problems (F. Magoules ed.). Saxe-Coburg Publications.

BANJAI, L. \& SAUTER, S. (2007) A refined Galerkin error and stability analysis for highly indefinite variational problems. SIAM J. Numer. Anal., 45, 37-53 (electronic).

Betcke, T., Chandler-Wilde, S. N., Graham, I. G., Langdon, S. \& Lindner, M. (2011) Condition number estimates for combined potential operators in acoustics and their boundary element discretisation. Numerical Methods for PDEs, 27, 31-69.

Betcke, T., Phillips, J. \& SPEnCE, E. A. (2012) Spectral decompositions and nonnormality of boundary integral operators in acoustic scattering - extended version. Technical Report. University College London.

\footnotetext{
${ }^{4} \mathrm{~A}$ proof of this statement was recently given to the authors by W. Wendland in a private communication: The fact that the double layer potential on the circle is of order -3 follows from the expansion (Hsiao \& Wendland, 2008, (2.1.20)) (essentially the low frequency asymptotics); the result for $C^{\infty}$ boundary $\Gamma$ follows by mapping the circle to $\Gamma$ using a $C^{\infty}$ differomorphism.
} 
Betcke, T. \& SPEnCE, E. A. (2011) Numerical estimation of coercivity constants for boundary integral operators in acoustic scattering. SIAM J. Numer. Anal., 49, 1572-1601.

Buffa, A. \& Hiptmair, R. (2005) Regularized combined field integral equations. Numerische Mathematik, 100, 1-19.

CEA, J. (1964) Approximation variationnelle des problèmes aux limites. Annales de L'Institut Fourier, 14, 345-444.

Chandler-Wilde, S. N., Graham, I. G., Langdon, S. \& Spence, E. A. (2012) Numericalasymptotic boundary integral methods in high-frequency scattering. Acta Numerica, 21, 89-305.

Chandler-Wilde, S. N. \& Langdon, S. (2007) A Galerkin Boundary Element Method for High Frequency Scattering by Convex Polygons. SIAM J. Numer. Anal., 45, 610-640.

Colton, D. L. \& Kress, R. (1983) Integral equation methods in scattering theory. Pure and Applied Mathematics (New York). New York: John Wiley \& Sons Inc., pp. xii+271. A Wiley-Interscience Publication.

Colton, D. \& KRess, R. (1998) Inverse acoustic and electromagnetic scattering theory. Springer.

Conway, J. B. (1985) A course in functional analysis. Graduate Texts in Mathematics, vol. 96. New York: Springer-Verlag.

Demmel, J. W. (1997) Applied numerical linear algebra. Philadelphia, PA: Society for Industrial and Applied Mathematics (SIAM), pp. xii+419.

Domínguez, V., Graham, I. G. \& Smyshlyaev, V. P. (2007) A hybrid numerical-asymptotic boundary integral method for high-frequency acoustic scattering. Numer. Math., 106, 471-510.

Engleder, S. \& Steinbach, O. (2007) Modified boundary integral formulations for the Helmholtz equation. Journal of Mathematical Analysis and Applications, 331, 396-407.

Engleder, S. \& Steinbach, O. (2008) Stabilized boundary element methods for exterior Helmholtz problems. Numerische Mathematik, 110, 145-160.

Gilbarg, D. \& Trudinger, N. (1998) Elliptic partial differential equations of second order, 2nd edn. Springer Verlag.

Gustafson, K. E. \& RaO, D. K. M. (1997) Numerical range. New York: Springer-Verlag. The field of values of linear operators and matrices.

HsiaO, G. C. \& Wendland, W. L. (2008) Boundary integral equations. Springer Verlag.

Kato, T. (1995) Perturbation Theory for Linear Operators. Springer.

KRESS, R. (1985) Minimizing the condition number of boundary integral operators in acoustic and electromagnetic scattering. The Quarterly Journal of Mechanics and Applied Mathematics, 38, 323.

KRESS, R. \& SPASSOV, W. T. (1983) On the condition number of boundary integral operators for the exterior Dirichlet problem for the Helmholtz equation. Numer. Math., 42, 77-95.

MCLEAN, W. (2000) Strongly Elliptic Systems and Boundary Integral Equations. Cambridge University Press.

Meyer, Y. \& CoIfman, R. (2000) Wavelets: Calderón-Zygmund and multilinear operators. Cambridge Studies in Advanced Mathematics. Cambridge University Press.

MitreA, M. (1996) Boundary value problems and Hardy spaces associated to the Helmholtz equation in Lipschitz domains. Journal of Mathematical Analysis and Applications, 202, 819-842. 
Monk, P. (2003) Finite element methods for Maxwell's equations. Oxford University Press, USA.

MoOn, P. \& SPENCER, D. E. (1971) Field theory handbook: including coordinate systems, differential equations and their solutions, 2nd edn. Springer-Verlag.

Morse, P. M. C. \& Feshbach, H. (1953a) Methods of Theoretical Physics, vol. 2. McGraw-Hill Science/Engineering/Math.

Morse, P. M. C. \& Feshbach, H. (1953b) Methods of Theoretical Physics, vol. 1. McGraw-Hill Science/Engineering/Math.

NIST (2011). Digital Library of Mathematical Functions, http://dlmf . nist . gov/.

PANICH, O. I. (1965) On the question of the solvability of exterior boundary-value problems for the wave equation and for a system of Maxwell's equations [in russian]. Uspekhi Mat. Nauk, 20:1(121), 221-226.

RAmm, A. G. (1973) Eigenfunction Expansion Associated with Discrete Spectrum in Problems of Diffraction. Radiotekh Elektron+, 18, 496-501.

Ramm, A. G. (1980) Nonselfadjoint operators in diffraction and scattering. Math. Methods Appl. Sci., 2, $327-346$.

RAnsford, T. (1995) Potential theory in the complex plane. Cambridge University Press.

REICHEL, W. (1997) Radial symmetry for elliptic boundary-value problems on exterior domains. Archive for Rational Mechanics and Analysis, 137, 381-394.

Rodin, G. J. \& Steinbach, O. (2003) Boundary element preconditioners for problems defined on slender domains. SIAM Journal on Scientific Computing, 24, 1450-1464 (electronic).

SERRIN, J. (1971) A symmetry problem in potential theory. Archive for Rational Mechanics and Analysis, 43, 304-318.

SloAn, I. H. (1992) Error analysis of boundary integral methods. Acta Numer. Cambridge: Cambridge Univ. Press, pp. 287-339.

Spence, E. A., Chandler-Wilde, S. N., Graham, I. G. \& Smyshlyaev, V. P. (2011) A new frequency-uniform coercive boundary integral equation for acoustic scattering. Communications on Pure and Applied Mathematics, 64, 1384-1415.

Spence, E. A., Kamotski, I. V. \& SmyshlyaeV, V. P. (2012) Coercivity of combined boundary integral equations in high frequency scattering. in preparation.

Trefethen, L. N. \& Embree, M. (2005) Spectra and pseudospectra. Princeton, NJ: Princeton University Press.

WARNICK, K. F. \& CHEW, W. C. (2001) On the spectrum of the electric field integral equation and the convergence of the moment method. Internat. J. Numer. Methods Engrg., 51, 31-56.

Wright, T. G. (2002). EigTool, http://wWw. comlab.ox.ac.uk/pseudospectra/eigtool. 